Sumiyana, S. (2020). Different characteristics of the aggregate of accounting earnings between developed and developing countries: Evidence for predicting future GDP. Journal of International Studies, 13(1), 58-80. doi:10.14254/2071$8330.2020 / 13-1 / 4$

\title{
Different characteristics of the aggregate of accounting earnings between developed and developing countries: Evidence for predicting future GDP
}

\author{
Sumiyana Sumiyana \\ Department of Accounting, Faculty of Economics and Business, \\ University of Gadjah Mada, \\ Jogyakarta, Indonesia \\ sumiyana@ugm.ac.id \\ ORCID 0000-0002-1518-9681
}

\begin{abstract}
This study investigates the relationship between the aggregate of accounting earnings and the future Gross Domestic Product (GDP) differentiating between developed and developing countries. This study employs data on Asian, African, and Pacific countries along with their capital market in the period of 1989-2015. More specifically, it investigates the informativeness of aggregate accounting earnings as a predictor of macroeconomic growth. It finds evidence that the aggregate of accounting earnings is a predictor of future GDP growth. It also shows that the informativeness of accounting earnings aggregate is not only for the capital market's level but also the macroeconomic level. This study argues that the informativeness of accounting earnings can be used to predict the macroeconomy, but only for those developed countries whose earnings growth is positive. On the contrary, this study suggests that the aggregate of accounting earnings in Asian and African developing countries cannot be used to predict future GDP growth. In other words, the aggregate of accounting earnings from developing countries does not contain similar properties for predicting future GDP growth as compared to those in developed countries.
\end{abstract}

Keywords: accounting earnings, gross domestic product, developing countries, future growth.

JEL Classification: M21, O16, P44

\section{INTRODUCTION}

The issue studied in this article is about the role of accounting earnings at the firms' level and their influence on macroeconomy. This relationship is rarely examined by accounting researchers (Konchitchki \& Patatoukas, 2014), although Beaver (1981), Kothari (2001), and Ball, Sadka, and Sadka (2009) have raised it before. This study investigates the relationship between the aggregate of accounting earnings and 
GDP for both developed and developing countries in the international setting. It takes into account that the characteristics of accounting earnings aggregate are different between developing countries and developed ones. At the same time, this study complements Sumiyana, Atmini, and Sugiri (2019) from another point of view due to the characteristics of the accounting earnings aggregate.

This study provides new evidence on the informativeness of accounting earnings as a predictor of economic growth that differentiates between developed and developing countries in the international setting. Researchers believe that GDP is an indicator of economic activities, including investments, production inputs, and labor absorption (Konchitchki \& Patatoukas, 2014). GDP is a statistical summary of those indicators (Bureau, 2007). The economists have heavily concentrated on GDP and its growth (Zarnowitz \& Braun, 1994). However, economics and accounting researchers do not, as yet, relate aggregate accounting earnings to GDP's growth (Konchitchki \& Patatoukas, 2014; Sumiyana et al., 2019). So, this study is useful for decision-makers, as well as for countries' central banks, national development bodies, and the World Bank. For developing countries, this study can be used to predict their future budgets, monetary policies, fiscal, and capital markets policies. Furthermore, it improves the prior association concept (Konchitchki \& Patatoukas, 2014) by specifying the changes in aggregate accounting earnings from period to period. This study aligns the changes in aggregate accounting earnings over time with the GDP's growth because it represents an actual change over time.

This study posits Konchitchki and Patatoukas (2014), Beaver (1981), Kothari (2001), and Ball et al. (2009). These studies argue that GDP's measurement and its growth align with the measurements of firms' accounting earnings, which have been calculated through an income approach known as the concept of capital maintenance (Kieso, Weygandt, \& Warfield, 2010). This income approach measures a firm's profits, managers' and employees' compensation, and taxes from every value-added item of production and import. Its measurements refer to each country's Generally Accepted Accounting Principles (GAAP). Meanwhile, the concept of the income approach serves as a measurement of firms' expected future cash flows. It confirms the GDP's concept, which depicts the potential of future valueadded flows. Thus, if the firms' accounting earnings are aggregated nationally, value-added creation can be measured nationally through this aggregation. Furthermore, the value-added creation is generated from the GPD and its growth (Konchitchki \& Patatoukas, 2014).

This study employs some assumptions. Firstly, the representativeness of the firms listed in a country's capital market is a reflection of that nation's macroeconomy. This assumption is also usable for developing countries, even though they may have only a few companies listed in their capital markets. It differs from companies in developed countries, where most of them are listed on the capital market. However, this assumption still applies, as private firms usually become the suppliers and customers of public companies, and vice versa. Secondly, all the listed firms in a country's capital market have utilitarianism ethics at the root of their business philosophy, which aims to increase the nation's welfare. This assumption also formulates that all the firms intend to move forward on a self-interest basis collectively.

Thirdly, this research did not address investors' protection, weak-law enforcement, and lessdeveloped capital markets (Porta, Florencio Lopez- de- Silanes, Andrei Shleifer, \& Robert W. Vishny, 1998; Rafael, Florencio, \& Andrei, 2006), or the low quality of the accounting information and the poor information environment (Leuz, Nanda, \& Wysocki, 2003). This study uses this assumption because of the inability to evaluate the accounting earnings' reliability and validity. This study notes the fact that some Asian and African countries were not mentioned in the research by Porta et al. (1998), and Rafael et al. (2006). Fourthly, this study uses a linearity assumption of the relationship between accounting earnings' profits and future predicted GDP. This linearity is based on a belief that there would be information contents in the aggregate accountings' earnings for the national macroeconomy. Lastly, the author believes 
that both the GDP's growth and the accounting numbers contain a high disclosure validity. In other words, this study accepts those two numbers.

This study uses established theories and prior studies. Ball et al. (2009) theorize that accounting earnings contain a concept of systematic components. This concept shows that accounting earnings at the firm level present information contents into the capital market. Furthermore, it presents this information content for predicting GDP aggregately. Ball et al. (2009) argument has been formulated by Kothari (2001), who suggests that there is a strong association between accounting earnings and market returns' volatilities. Thus, according to Ball et al. (2009) and Kothari (2001) theories, this study ties together capital markets, foreign exchange reserves, monetary policies, and fiscal policy related theories. Gonedes (1973b) argues that accounting earnings growth can lever foreign exchange reserves in the next year. Likewise, Taylor (1993) and Clarida, Gali, and Gertler (2000) conclude that an increase in accounting earnings is usually supported by monetary policies, as well as fiscal policies, for the next few years. Moreover, Landefeld, Seskin, and Fraumeni (2008), Newey and West (1987), and Bureau (2007) find a similar conclusion.

This study contributes to studies in accounting and the macroeconomy. The details of this study's contributions are as follows: Firstly, these study associates aggregate accounting earnings and future GDP's growth in an international setting. This setting distinguishes this association with developed and developing countries explicitly. Moreover, the study focuses on the Asia-Pacific region and Africa, because most of the developing countries are in those regions. The aggregate of accounting earnings at the firms' level, as well as their growth in developing countries, should also play a role as an indicator of future GDP. Consequently, professional forecasters of macro economies are advised to include accounting earnings as a GDP growth predictor. On the other hand, the informativeness of accounting earnings does not only pay court to the capital market but also applies to a nation's macroeconomy. It shows that the "micro to micro" relationship, which explains the relationship between accounting earnings and market returns, does not stop there. The relationship that explains the aggregate of accounting earnings and the national macroeconomy has become a new phenomenon (Konchitchki \& Patatoukas, 2014). Therefore, it can be said that this study extends the informativeness of the aggregate of accounting earnings and the methodological improvement of prior studies.

Secondly, this study improves the prior concept developed by Konchitchki and Patatoukas (2014). The improvement transforms the aggregate accounting earnings into the change in aggregate accounting earnings. This study ultimately matches the change in aggregate accounting earnings with GDP growth. Therefore, it matches perfectly. In other words, this research equalizes the measurement concepts for both the GDP's growth and the change in aggregate accounting's earnings.

This study is structured as follows. Part 2 highlights the literature review and hypotheses development. It focused on the informativeness of accounting earnings as a predictor of economic growth that differentiates between developed and developing countries in an international setting. Part 3 thoroughly explains the research method used in this study, ranging from the sampling to the hypotheses testing. This research designed a new methodological refinement. It, specifically, induced the predictive consensus towards the GDP's predictive elements. Part 4 discusses the descriptive statistics, analysis, and findings. This research inferred that the associations between aggregate accounting earnings and GDP growths could not be equivalenced among developed and developing countries due to differential properties. Part 5 summarises the conclusions and suggestions and the possibilities for future research. It concluded that changes in accounting earnings are not a reliable predictor for future GDP growth in developing countries. It concluded that accounting numbers do not adequately reflect economic productivity in developing countries, due to future economic uncertainties relative to those of the developed countries. 


\section{LITERATURE REVIEW}

\subsection{Accounting earnings}

Gonedes (1973b) formulates that accounting earnings (accounting numbers) are suggested to have a keen ability to influence the capital market, more commonly known as an economy-wide ability. In this regard, accounting earnings can widely influence the capital market (Brown \& Ball, 1967; Gonedes, 1973b). Accounting earnings at the firms' level influence the capital markets through similar industries and influence the national economy of a country, although the influence can be formed reversely. These previous three studies started to identify accounting earnings' properties by relating them to their ability to influence an economy extensively. Therefore, this study shows that accounting earnings, the capital market's rate of return, the industry-wide regime, and the national economy, are accompanied by each other.

Prior studies have examined the influence of accounting earnings. Firstly, they investigated the influence of accounting earnings on share prices using firm-specific accounting earnings. In this phase, the prior studies employed firm-specific accounting earning's properties to influence share prices, and this is known as the firm-specific approach (Basu, 1977; Conrad, Cornell, \& Landsman, 2000; Dechow, Kothari, \& L. Watts, 1998; Lopez \& L. Rees, 2001; Skinner \& Sloan, 2002). Most studies related to accounting earnings have been developed to not only use accounting earnings individually but also employ broader economic influences, known as the market-wide regime shifting behavior (Ho \& Sequeira, 2007). Nevertheless, prior studies related to this market-wide behavior have been designed by Brown and Ball (1967), Gonedes (1973b) and Kothari, Lewellen, and Warner (2006). The influence of accounting earnings in this regard includes the capital market's movements and the nation's macroeconomy, which shows that its influences have been developing widely at the national economic level, and vice versa.

The influence of accounting earnings' properties on a national macroeconomy has been continuously studied by some researchers, such as Konchitchki and Patatoukas (2014) and Patatoukas (2014). In this phase, the level of study is not on the individual level of accounting earnings' properties, but on the national aggregate of accounting earnings' properties. Furthermore, accounting earnings influence monetary policies, foreign exchange policies, fiscal policies and the macroeconomy as a whole. The results of these two studies show that the aggregate of accounting earnings influences the national macroeconomy's climate. It also shows that the aggregate of accounting earnings has informativeness not only at the firms' level but also at the market-wide and nation-wide levels.

\subsection{GDP and its growth}

To date, economists still believe that GDP and its growth is the measure of national revenue and social welfare (Fleurbaey, 2009; Fleurbaey \& Gaulier, 2009; Henderson, Storeygard, \& Weil, 2012). GDP measures income from all the entities and households involved in economic activities within a country. Meanwhile, these studies also argue that GDP and its growth are comprehensive indicators that can reliably measure national income. On the other hand, Becker, Philipson, and Soares (2005) argue that it has a function of consumption utility, which can also be seen (from the opposite view) as a function of income utility. Related to its function as consumption and income measurement, GDP might be seen as a productivity measurement, reflecting what has been achieved by all the firms and households nationally. Thus, GDP primarily measures the performance of the economic activities of a country.

This study temporarily concludes that GDP is the total of the consumption and income of a country. Meanwhile, accounting earnings are the income of a company, and if they are the total of all the firms in the capital market, then this is called the GDP. It is consistent with the arguments proposed by Becker et 
al. (2005). Furthermore, following the same argument, these studies argue that the total amount of the growth in firms' accounting earnings from year-to-year reflects the GDP's growth from year-to-year. However, this study acknowledges that these total numbers are only partial as all the firms are not listed on the capital market.

\subsection{Accounting earnings and future GDP growth}

McCloskey (1993) argues that accounting logic relates to a country's accounting logic, especially in terms of its national income. He explicitly argues that firms pay employees' wages, which will be consumed. If there is an amount left over from their wages, they may save it in a savings account. Meanwhile, a country supplies money for the sake of firms and their employees. Thus, McCloskey (1993) concludes that either its expenses or income reflect the life of a firm. Therefore this is a reflection of the national income of a country.

Moreover, accounting and financial management researchers (for example, Fama (1981)) have also argued that share prices or returns from the capital market reflect the inflation level and the money supply. The congeniality of variance movement between share returns and the money supply, as well as inflation, is always harmonious (Fischer \& Merton, 1984). They state that the macroeconomy plays a role in improving the capital market and vice versa. A study related to the capital market, as well as the money market, was conducted by Harvey (1989). Harvey (1989) argues that economic growth is denominated by the capital market's development and the bond market. Similarly, Shevlin (1990) concludes that a country's revenue from firms' taxes shows the marginally increasing movement of revenue and all the firms' losses, within a country. These studies show that harmonious movements between the GDP and the total national income and the capital market are included in the money market. Either it is from the GDP or capital market, as well as the money market's point of view, that these two extremes determine the growth and improvement of each other.

Over the last decade, more in-depth studies have been undertaken by Kothari et al. (2006), Lev and Nissim (2004), and Ho and Sequeira (2007). They argue that earned returns do not merely come from the movement of the individual accounting earnings of a firm. Preferably, the movement of earned returns comes from national economic movements, while the capital market automatically levers national economic improvements. The concept of these studies changes the paradigm, from a firm-specific approach to a market-wide regime shifting behavior. It shows that the market has full control over increasing or decreasing investors' returns. In the end, firms earn higher profits by employing lower costs of capital. Otherwise, when a firm earns a higher profit, the capital market and money market increase widely. The increase provides investors and creditors with disposable income which subsequently increases the national income.

Some studies concluded that the macroeconomy could alter a firm's ability to earn a profit (Fischer \& Merton, 1984; Harvey, 1989; McCloskey, 1993). Moreover, other studies also provided evidence that firms' activities reflect accounting earnings' increases in the national income's growth as well as in the GDP (Ho \& Sequeira, 2007; Kothari et al., 2006; Lev \& Nissim, 2004). Thus, this preliminary study concludes that the firms' abilities to earn profits, and their economic activities, influence the capital and money markets. This dependency has become the basis for developing this study.

\subsection{Hypotheses developments}

This study argues that the aggregate of accounting earnings is used to predict future GDP growth. Prior studies argued that GDP's growth could be predicted not only through macroeconomic indicators, but also through the aggregate of the accounting earnings of all the listed firms on the capital market, 
which could be deemed as a good indicator (Konchitchki, 2011; Konchitchki \& Patatoukas, 2014; Sumiyana et al., 2019). This study has explored this and believed that the GDP's growth predictor does not as yet exist. Therefore, it proposes that the aggregate accounting numbers are used as the predictor of future GDP growth.

GDP and its growth reflect all the firms' consumption, as well as the households' consumption within a country. It also depicts that the GDP and its growth reflect all the firms' and households' income within a country (Bureau, 2002, 2004, 2007; Karnitis \& Karnitis, 2017; Oliinyk \& Kozmenko, 2019). Accounting earnings showing the difference between income and expenses should reflect the GDP and its growth (Bureau, 2002, 2004, 2007; Karnitis \& Karnitis, 2017; Shkolnyk, Kozmenko, Kozmenko, \& Mershchii, 2019; Tung, 2018). On the other hand, this study maintains and re-examines the concept developed by Konchitchki and Patatoukas (2014), and Sumiyana et al. (2019). This study argues that the aggregate accounting earnings of all the firms within a country fully reflects all the economic activities nationally. It posits the concepts of the "micro to macro" relationship. It means that all of the microeconomic activities are aggregated into the macro. This study also argues that the aggregate accounting earnings -by measurement- are the changes in revenues and expenses, and these correctly associate with GDP's growth. Thus, this study hypothesizes that the aggregate of the accounting earnings earned from a country's capital market could predict the GDP's growth. It constructs the hypothesis statement as follows:

$\mathbf{H}_{\mathbf{A 1}}$ : The aggregate of accounting earnings positively associates with the GDP's growth.

This study furthermore develops the association concept Konchitchki and Patatoukas (2014) by specifying the change in aggregate accounting earnings from period to period. This study argues that GDP's growth is also a change over periods so that this study aligns with the changes in aggregate accounting earnings over time. Thus, this study hypothesizes that the changes in aggregate accounting earnings, earned by a country's capital market, could predict the GDP's growth. Then, this study constructs the following hypothesis statement:

$\mathbf{H}_{\mathrm{A} 2}$ : The changes in the aggregate of the accounting earnings positively associate with the GDP's growth.

\section{METHODOLOGY}

\subsection{Sample and unit analysis}

This study obtained accounting earnings information and other fundamental information from the OSIRIS database. Yearly data for the aggregate accounting earnings, assets, liabilities, equities, revenues, and expenses were obtained from every capital market within a country, ranging from the year the capital market was established to 2016. This study employs capital market data from Asia-Pacific and African countries, as they are categorized as being underdeveloped or developing countries. Furthermore, predicted and future GDP growth is obtained from the central bank within each country.within a country if any. If no data are found, this study analyzed yearly future predicted GDP deviations (Konchitchki \& Patatoukas, 2014; Landefeld et al., 2008; Romer \& Romer, 2000). Data related to GDP's properties are also collected from the World Bank and its website.

This study employs a national-year unit analysis. National refers to the aggregate of the accounting earnings by totaling all the profits of all the listed companies in the capital market within a country. The 
calculation process is complemented by scaling changes to the aggregate of accounting earnings over the previous year. Year refers to the yearly total accounting earnings of the capital market in each country. Therefore, the timeline of this study uses the aggregate of the accounting earnings of all the listed companies in the capital markets of every country from year to year.

\subsection{Variables and measurements}

This study uses three main variables, i.e., the aggregate of accounting earnings (X), GDP growth (g), and the error variance of the predicted GDP (E). About the unit analysis, the variables are defined as the aggregate of the accounting earnings $\left(\mathrm{X}_{\mathrm{it}}\right)$, GDP growth $\left(\mathrm{g}_{\mathrm{it}}\right)$, and the variance error of the predicted GDP (it), while " $i$ " represents the national level, ' $\mathrm{t}$ " represents the year. Moreover, this study calculates the aggregate of the accounting earnings growth $\left(\Delta \mathrm{X}_{\mathrm{it}}\right)$ by subtracting the current earnings from last year's earnings $\left(\mathrm{X}_{\mathrm{it}}-\mathrm{X}_{\mathrm{it}-1}\right)$. This accounting earnings growth is scaled by the aggregate of the total asset $\left(\mathrm{A}_{\mathrm{it}}\right)$, the aggregate of total debt $\left(\mathrm{L}_{\mathrm{it}}\right)$, the aggregate of total revenue $\left(\mathrm{R}_{\mathrm{it}}\right)$ or the last year's $(\mathrm{t}-1)$ aggregate of the shares' book value $\left(B_{i t}\right)$ of all the listed companies. This scale is formulated to eliminate the size bias of the capital market from every country.

This study provides a link between the aggregate of the accounting earnings and the next year's predicted GDP growth $\left(g_{i t+1}\right)$. In determining the value of the predicted GDP growth $\left(g_{i t+1}\right)$, researchers have agreed on a consensus, in which the predicted growth has to be adjusted with next year's predicted error variance $\left(\mathrm{e}_{\mathrm{it}+1}\right)$ (A. Sims, 2002; Abarbanell, 1991; Ang, Bekaert, \& Wei, 2007; Ang, Piazzesi, \& Wei, 2006; Baghestani \& M Kianian, 1993; Bradshaw, Drake, Myers, \& Myers, 2012; Chung \& Kryzanowski, 1999; Darrough, 2002; Zarnowitz \& Braun, 1994). The consensus agrees that an adjusted valued $\left(g_{i t+1} \cdot e_{i t+1}\right)$ is used for decreasing or increasing the adjusted GDP growth $\left(g_{i t+k}\right)$. Therefore, the GDP growth used to predict its future should be $\left(g_{i t+1}\right)$ for itself and $\left(g_{i t+1} \cdot e_{i t+1}\right)$. This study defines predicted growth not only by predicting one year forward (lead), but also predicting the GDP with a two years' lead, which is ( $\left.g_{i t+k}\right)$ and $\left(\mathrm{g}_{\mathrm{it}+\mathrm{k}} \cdot \mathrm{e}_{\mathrm{it}+\mathrm{k}}\right)$, while $\mathrm{k}$ represents 1 and 2.

\subsection{Hypotheses Testing}

This study constructs several regression models. Firstly, it examines the association between the next year's predicted GDP and the aggregate of the current accounting earnings. This test acts as an initial identification that the aggregate of the accounting earnings can predict the GDP's growth. The regression model is as follows:

$$
g_{i t+k}=\alpha_{k}+\beta_{k} X_{i t}+e_{i t+k}
$$

Therefore, git +1 changes on a yearly differentiation and this study equalize the second regression model with the changes in the earnings aggregate differentiation $\Delta \mathrm{X}_{\mathrm{it}}$. The second regression model is as follows:

$$
g_{i t+k}=\alpha_{k}+\beta_{k} \Delta X_{i t}+e_{i t+k}
$$

Moreover, this study considers the predictive consensus towards the GDP's growth. The next test includes the previous GDP's predictive elements (A. Sims, 2002; Abarbanell, 1991; Ang et al., 2007; Ang et al., 2006; Baghestani \& M Kianian, 1993; Bradshaw et al., 2012; Chung \& Kryzanowski, 1999; Darrough, 2002; Zarnowitz \& Braun, 1994). Thus, the third, fourth, and fifth regression models are respectively as follows:

$$
g_{i t+k}=\alpha_{k}+\beta_{k} \Delta X_{i t}+\delta_{k} g_{i t+(k-1)}+e_{i t+k}
$$




$$
\begin{aligned}
& g_{i t+k}=\alpha_{k}+\beta_{k} \Delta X_{i t}+\varphi_{k} g_{i t+(k-1)} \cdot e_{i t+k}+e_{i t+k} \\
& g_{i t+k}=\alpha_{k}+\beta_{k} \Delta X_{i t}+\delta_{k} g_{i t+(k-1)}+\varphi_{k} g_{i t+(k-1)} \cdot e_{i t+k}+e_{i t+k}
\end{aligned}
$$

All the regression models are within the linear regression boundary. Therefore, this study complements the linearity testing to decrease the error bias by employing normality, heteroscedasticity, and multicollinearity (White, 1980). The objectives of these tests are to ensure that the regression models are linear and have no dominant error bias (White, 1980). The hypotheses testing places specific emphasis on the coefficient of $\beta_{k} \neq 0$ and $\beta_{k}>0$ to answer the direction and the strength of the influence of the earnings aggregate on the GDP's growth. These tests use a degree of freedom $(\alpha)$ of $10.00 \%, 5.00 \%$, and $1.00 \%$, respectively.

\subsection{Sensitivity test}

This study complements its validity of the conclusions. It makes reexamination designs by separating the samples into several area clusters. Separation of the samples is exercised country-to-country; the groups of countries include Southeast Asia, South Asia, and East Asia, as well as the group of continents, which are Asia and Africa. This study argues that the GDP's growth behavior tends to be similar for countries in a particular area cluster. Furthermore, it makes a cut-off separation from year-to-year, particularly in the years of the global financial crises which occurred in 1997-1998 and 2009-2010. The separation, either by excluding years or cutting-off the annual period, is to show the effect of the global financial crises on accounting earnings. More specifically, the error of predictive bias had substantially increased its influence while the world's economy decreased drastically.

\section{EMPIRICAL RESULTS AND DISCUSSION}

\subsection{Descriptive statistics}

This study identifies 71 Asian and African countries around the South China Sea and the Atlantic Ocean. However, only 39 Asia-Pacific and African countries formed our sample as there is no capital market information, earnings aggregates, or GDP growth data for the other 32 countries. Those countries are American Samoa, Benin, Burkina Faso, Bangladesh, Brunei Darussalam, Bhutan, Federated States of Micronesia, Gabon, The Gambia, Guam, Cambodia, Kiribati, Liberia, Marshall Islands, Myanmar, Mongolia, Northern Mariana Islands, New Caledonia, Niger, Nauru, Palau, Republic of Korea's Democratic People, French Polynesia, Rwanda, Senegal Solomon Islands, Togo, Timor Leste, Tonga, Tuvalu, Vanuatu, and Zimbabwe. This study also separated the developed countries from the sample, such as Australia, New Zealand, China, Hong Kong, Japan, Singapore, South Korea, and Taiwan. Thus, this study employs 39 Asia-Pacific and African countries covering the years from 1989-2015.

Descriptive statistics analysis shows the earnings aggregate, along with the descriptive data and GDP growth in Table 1 Panel A - H. From the grouped nations' data, this study summarises the descriptive statistics for the sample. This study separates the sample into two groups, i.e., developed and developing countries. Then, the developing countries are separated into two further groups, i.e., Asian and African countries. This study also separates the sample into three periods, i.e., 1989-1997, 2000-2007, and 20102015. 
Table 1

Panel A: All Sample (n: 584)

\section{Descriptive statistics}

\begin{tabular}{|c|c|c|c|c|c|c|c|c|c|}
\hline Description & Notation & Min. & Max. & Mean & Std. Dev. & Skewness & Perc. -25 & Perc. -50 & Perc. -75 \\
\hline Agregate Earnings & $X_{i t}$ & 30.09 & $283,080,562.20$ & $15,175,154.33$ & $37,857,342.90$ & 4.0976 & $97,944.26$ & $805,931.61$ & $9,109,030.67$ \\
\hline$\Delta$ Agregate Earnings & $x_{i t}-x_{i t+(k+1)}$ & $106,896,959.20$ & $106,945,588.00$ & $1,753,239.11$ & $9,167,522.26$ & 1.2361 & -864.45 & $51,022.08$ & $861,777.05$ \\
\hline$\Delta$ Agregate Earnings $(\%)$ & $\begin{array}{c}\left(x_{i t}-\right. \\
\left.x_{i t+(k+1)}\right) / x_{i l+(k+1)} \\
\end{array}$ & -0.9994 & $7,071.5720$ & 16.0263 & 303.4927 & 22.0773 & -0.0144 & 0.2036 & 0.5227 \\
\hline GDP Growth & $g_{i t}$ & -52.5780 & 27.9288 & 0.0952 & 4.7774 & -4.4811 & -0.3771 & -0.0128 & 0.4006 \\
\hline GDP Growth (t-1) & $g_{i t+(k+1)}$ & -52.5780 & 37.9351 & 0.0434 & 4.4380 & -3.0585 & -0.4456 & -0.0475 & 0.3538 \\
\hline
\end{tabular}

Panel B: Developed Countries' Sample (n: 179)

\begin{tabular}{|c|c|c|c|c|c|c|c|c|c|}
\hline Description & Notation & Min. & Max. & Mean & Std. Dev. & Skewness & Perc. -25 & Perc. -50 & Perc. -75 \\
\hline Agregate Earnings & $X_{i t}$ & $25,064.36$ & $283,080,562.20$ & $39,911,582.99$ & $58,761,988.55$ & 2.2324 & $2,775,468.28$ & $13,954,667.93$ & $55,163,609.68$ \\
\hline$\Delta$ Agregate Earnings & $x_{i t}-x_{i t+(k+1)}$ & $-23,952,712.14$ & $59,659,621.47$ & $4,879,724.63$ & $11,053,932.70$ & 1.9276 & $11,555.75$ & $953,955.17$ & $8,805,843.22$ \\
\hline$\Delta$ Agregate Earnings $(\%)$ & $\begin{array}{c}\left(x_{i t}-\right. \\
\left.x_{i t+(k+1)}\right) / x_{i l+(k+1)} \\
\end{array}$ & -0.7813 & 18.0231 & 0.4408 & 1.5052 & 9.4183 & 0.0108 & 0.1854 & 0.4540 \\
\hline GDP Growth & $g_{i t}$ & -52.5780 & 9.1756 & -0.1075 & 5.1972 & -7.1461 & -0.4495 & -0.0603 & 0.5001 \\
\hline GDP Growth (t-1) & $g_{i+(k+1)}$ & -52.5780 & 14.3333 & -0.2483 & 5.2403 & -6.8156 & -0.5347 & -0.1117 & 0.3708 \\
\hline
\end{tabular}

Panel C: Developing Countries' Sample (n: 405)

\begin{tabular}{|c|c|c|c|c|c|c|c|c|c|}
\hline Description & Notation & Min. & Max. & Mean & Std. Dev. & Skewness & Perc. -25 & Perc. -50 & Perc. -75 \\
\hline Agregate Earnings & $X_{i t}$ & 30.09 & $106,960,711.30$ & $4,242,263.64$ & $12,485,315.26$ & 4.7492 & $59,580.81$ & $244,742.95$ & $1,835,531.18$ \\
\hline$\Delta$ Agregate Earnings & $x_{i t}-x_{i t+(k+1)}$ & $106,896,959.20$ & $106,945,588.00$ & $371,409.71$ & $7,823,008.72$ & -0.0564 & $-2,119.63$ & $20,360.29$ & $213,249.08$ \\
\hline $\begin{array}{l}\Delta \text { Agregate Earnings } \\
(\%)\end{array}$ & $\begin{array}{c}\left(x_{i t}-\right. \\
\left.x_{i+1}(k+1)\right) / x_{i t+(k+1)} \\
\end{array}$ & -0.9994 & $7,071.5720$ & 22.9147 & 364.3649 & 18.3817 & -0.0342 & 0.2284 & 0.5303 \\
\hline GDP Growth & $g_{i t}$ & -46.0078 & 27.9288 & 0.1847 & 4.5835 & -2.7554 & -0.3484 & 0.0228 & 0.3822 \\
\hline GDP Growth (t-1) & $g_{i t+(k+1)}$ & -40.9294 & 37.9351 & 0.1723 & 4.0335 & 0.7216 & -0.3758 & -0.0191 & 0.3489 \\
\hline
\end{tabular}

Panel D: Asia's Sample (n: 181)

\begin{tabular}{|c|c|c|c|c|c|c|c|c|c|}
\hline Description & Notation & Min. & Max. & Mean & Std. Dev. & Skewness & Perc. -25 & Perc. -50 & Perc. -75 \\
\hline Agregate Earnings & $X_{i t}$ & 130.68 & $79,739,341.60$ & $8,200,025.27$ & $16,032,784.59$ & 2.8416 & $191,007.19$ & $1,227,066.43$ & $9,092,739.45$ \\
\hline$\Delta$ Agregate Earnings & $x_{i t}-x_{i t+(k+1)}$ & $-9,010,375.44$ & $18,394,863.74$ & $836,858.49$ & $2,999,626.62$ & 2.6606 & $5,102.08$ & $140,974.93$ & $931,435.10$ \\
\hline$\Delta$ Agregate Earnings $(\%)$ & $\begin{array}{c}\left(x_{i t}-\right. \\
\left.x_{i t+(k+1)}\right) / x_{i t+(k+1)} \\
\end{array}$ & -0.8560 & 26.5967 & 0.6014 & 2.4980 & 9.0178 & 0.0247 & 0.2753 & 0.5402 \\
\hline GDP Growth & $g_{i t}$ & -46.0078 & 27.9288 & -0.1229 & 6.4798 & -2.1850 & -0.3080 & 0.0046 & 0.2690 \\
\hline GDP Growth (t-1) & $g_{i t+(k+1)}$ & -40.9294 & 37.9351 & 0.0756 & 5.7214 & 0.5138 & -0.3171 & 0.0046 & 0.2998 \\
\hline
\end{tabular}

Panel E: Africa's Sample (n: 224)

\begin{tabular}{|c|c|c|c|c|c|c|c|c|c|}
\hline Description & Notation & Min. & Max. & Mean & Std. Dev. & Skewness & Perc. -25 & Perc. -50 & Perc. -75 \\
\hline Agregate Earnings & $X_{i t}$ & 30.09 & $106,960,711.30$ & $1,044,250.88$ & $7,206,643.91$ & 14.3738 & $30,906.96$ & $108,640.06$ & $428,650.04$ \\
\hline$\Delta$ Agregate Earnings & $x_{i t}-x_{i t+(k+1)}$ & $106,896,959.20$ & $106,945,588.00$ & $-4,689.53$ & $10,163,261.46$ & 0.0004 & $-4,033.92$ & $9,089.43$ & $54,924.64$ \\
\hline$\Delta$ Agregate Earnings $(\%)$ & $\begin{array}{c}\left(x_{i t}-\right. \\
\left.x_{i t+(k+1)}\right) / x_{i t+(k+1)} \\
\end{array}$ & -0.9994 & $7,071.5720$ & 40.9447 & 489.6769 & 13.6634 & -0.08 & 0.17 & 0.53 \\
\hline GDP Growth & $g_{i t}$ & -5.6902 & 11.5274 & 0.4333 & 2.0075 & 3.0566 & -0.3771 & 0.0567 & 0.5634 \\
\hline GDP Growth (t-1) & $g_{i t+(k+1)}$ & -5.6902 & 10.8358 & 0.2504 & 1.7430 & 2.9534 & -0.3950 & -0.0433 & 0.3942 \\
\hline
\end{tabular}

Panel F: Split Sample for the period of 1989-1996 (n: 104)

\begin{tabular}{|l|c|r|r|r|r|r|r|r|r|}
\hline \multicolumn{1}{|c|}{ Description } & Notation & \multicolumn{1}{c|}{ Min. } & \multicolumn{1}{c|}{ Max. } & \multicolumn{1}{c|}{ Mean } & \multicolumn{1}{c|}{ Std. Dev. } & \multicolumn{1}{c|}{ Skewness } & \multicolumn{1}{c|}{ Perc. -25 } & \multicolumn{1}{c|}{ Perc. -50 } & \multicolumn{1}{c|}{ Perc. - 75 } \\
\hline Agregate Earnings & \multicolumn{1}{c|}{$X_{i t}$} & 130.68 & $40,863,147.44$ & $3,504,978.84$ & $6,602,741.04$ & 3.3098 & $243,567.90$ & $819,731.42$ & $3,833,826.90$ \\
\hline$\Delta$ Agregate Earnings & $x_{i t}-x_{i+(k+1)}$ & $-5,131,399.09$ & $13,227,828.24$ & $669,276.16$ & $2,152,203.25$ & 3.7894 & $23,605.02$ & $179,048.37$ & $561,604.50$ \\
\hline$\Delta$ Agregate Earnings (\%) & $\begin{array}{c}\left(x_{i t}-\right. \\
x_{i t+(k+1)} / x_{i+(k+1)}\end{array}$ & -0.8560 & 20.1250 & 0.6684 & 2.1035 & 8.0121 & 0.0948 & 0.3045 & 0.5920 \\
\hline GDP Growth & $g_{i t}$ & -2.1341 & 16.3158 & 0.9018 & 3.1787 & 2.9864 & -0.5099 & 0.0046 & 0.4847 \\
\hline GDP Growth (t-1) & $g_{i t+(k+1)}$ & -2.1341 & 12.8586 & 0.5967 & 2.4688 & 3.4297 & -0.4533 & 0.0270 & 0.5178 \\
\hline
\end{tabular}


Panel G: Split Sample for the period of 1999-2007 (n: 265)

\begin{tabular}{|c|c|c|c|c|c|c|c|c|c|}
\hline Description & Notation & Min. & Max. & Mean & Std. Dev. & Skewness & Perc. -25 & Perc. -50 & Perc. -75 \\
\hline Agregate Earnings & $X_{i t}$ & 30.09 & $203,863,970.40$ & $10,585,173.21$ & $26,108,096.49$ & 4.5349 & $59,580.81$ & $661,856.63$ & $9,107,045.92$ \\
\hline$\Delta$ Agregate Earnings & $x_{i t}-x_{i t+(k+1)}$ & $106,896,959.20$ & $106,945,588.00$ & $1,976,628.92$ & $11,046,767.84$ & 0.1094 & $3,688.25$ & $81,007.76$ & $1,353,020.25$ \\
\hline$\Delta$ Agregate Earnings $(\%)$ & $\begin{array}{c}\left(x_{i t}-\right. \\
\left.x_{i t+(k+1)}\right) / x_{i t+(k+1)} \\
\end{array}$ & -0.9994 & $7,071.5720$ & 34.8481 & 450.2768 & 14.8642 & 0.0571 & 0.3199 & 0.7375 \\
\hline GDP Growth & $g_{i t}$ & -52.5780 & 27.9288 & -0.0885 & 6.7499 & -3.5929 & -0.3586 & 0.1022 & 0.7349 \\
\hline GDP Growth (t-1) & $g_{i t+(k+1)}$ & -52.5780 & 37.9351 & -0.0209 & 6.3521 & -2.3754 & -0.4242 & 0.0360 & 0.5510 \\
\hline
\end{tabular}

Panel H: Split Sample for the period of 2010-2015 (n: 215)

\begin{tabular}{|c|c|c|c|c|c|c|c|c|c|}
\hline Description & Notation & Min. & Max. & Mean & Std. Dev. & Skewness & Perc. -25 & Perc. -50 & Perc. -75 \\
\hline Agregate Earnings & $X_{i t}$ & $2,467.00$ & $283,080,562.20$ & $26,477,681.05$ & $53,121,658.65$ & 2.8308 & $121,517.08$ & $1,337,871.00$ & $24,609,024.43$ \\
\hline$\Delta$ Agregate Earnings & $x_{i t}-x_{i t+(k+1)}$ & $-17,067,702.42$ & $59,659,621.47$ & $2,002,233.75$ & $8,688,613.62$ & 3.4438 & $-37,413.05$ & $12,444.15$ & $503,163.22$ \\
\hline$\Delta$ Agregate Earnings $(\%)$ & $\begin{array}{c}\left(x_{i t}-\right. \\
\left.x_{i+1(k+1)}\right) / x_{i t+(k+1)} \\
\end{array}$ & -0.9855 & 26.5967 & 0.2562 & 1.8732 & 13.1875 & -0.0816 & 0.0618 & 0.2753 \\
\hline GDP Growth & $g_{i t}$ & -4.4734 & 5.0659 & -0.0686 & 0.8511 & 0.4322 & -0.3733 & -0.1097 & 0.1636 \\
\hline GDP Growth (t-1) & $g_{i t+(k+1)}$ & -4.4734 & 5.0659 & -0.1451 & 0.8703 & 0.4376 & -0.4486 & -0.1413 & 0.1308 \\
\hline
\end{tabular}

Note: Earnings aggregate, along with minimum, maximum, mean, and standard deviation is stated in US\$ million; Predicted GDP is stated in US\$ billion.

Inferences gained from Table 1 Panel B and C as a comparison between the developing countries and developed countries are as follows: The earnings aggregate growth of the developing countries is higher than that of the developed countries. It shows growth with a mean of $22.9147 \%$ for the developing countries and $0.4408 \%$ for the developed countries. A critical point that has to be noted is that the GDP's growth in developing countries grows with a mean of $0.1847 \%$. Contrary to the developed countries, it could grow below the zero points, with a mean of $-0.1075 \%$. However, under-zero growth is only experienced by Asian countries, with a mean of $-0.1229 \%$. This study notes that both the earnings aggregate and GDP growth have a positive skewness of 18.3817 for the developing countries and 9.4183 for the developed countries. This study infers that there are more countries with lower earnings aggregate and GDP growth than with a higher one, because of this positive skewness.

This study undertakes a correlation diagnosis among the variables in Table 2. It shows that almost all of the aggregate earnings, and their growth, do not correlate as being statistically significant to the GDP's growth in developing countries. There is no correlation between the aggregate earnings growth and GDP growth in all the panels. This study initially infers that both the numbers for aggregate earnings and aggregate earnings growth do not relate to the GDP's growth in developed and developing countries. It means that all of the hypotheses will not be supported in the subsequent regression analysis for the developed countries.

Table 2

Panel A: All Sample (n: 584)

Correlation diagnostics

\begin{tabular}{|c|c|c|c|c|c|c|c|c|}
\hline \multirow{2}{*}{\multicolumn{2}{|c|}{ All }} & \multirow{2}{*}{\multicolumn{2}{|c|}{$\frac{\text { Aggregate Earnings }}{X_{i t}}$}} & \multirow{2}{*}{\multicolumn{2}{|c|}{$\frac{\Delta \text { Agg. Earnings }}{x_{i t}-x_{i t+(k+1)}}$}} & \multirow{2}{*}{\begin{tabular}{|l|}
$\Delta$ Agg. Earnings $(\%)$ \\
$\left(x_{i t}-x_{i t+(k-1)}\right) / x_{i t+(k-1)}$
\end{tabular}} & \\
\hline & & & & & & & $g_{i t}$ & \\
\hline$\Delta$ Agregate Earnings & $x_{i t}-x_{i t+(k-1)}$ & 0.4560 & $* * *$ & & & & & \\
\hline$\Delta$ Agregate Earnings $(\%)$ & $\left(x_{i t}-x_{i t+(k-1)}\right) / x_{i t+(k-1)}$ & 0.0920 & ** & 0.4570 & $* * *$ & & & \\
\hline GDP Growth & $g_{i t}$ & 0.0213 & & 0.0295 & & 0.0026 & & \\
\hline GDP Growth (t-1) & $g_{i t+(k-1)}$ & 0.0091 & & 0.0350 & & 0.0019 & 0.7190 & *** \\
\hline
\end{tabular}


Panel B: Developed Countries' Sample (n: 179)

\begin{tabular}{|c|c|c|c|c|c|c|c|}
\hline \multirow{2}{*}{\multicolumn{2}{|c|}{ Developed }} & \multirow{2}{*}{\multicolumn{2}{|c|}{$\begin{array}{c}\text { Aggregate Earnings } \\
X_{i t}\end{array}$}} & \multirow{2}{*}{$\Delta$ Agg. Earnings } & \multirow{2}{*}{$\Delta$ Agg. Earnings (\%) } & \multirow{2}{*}{\multicolumn{2}{|c|}{ GDP growth }} \\
\hline & & & & & & & \\
\hline$\Delta$ Agregate Earnings & $x_{i t}-x_{i t+(k-1)}$ & 0.4970 & $* * *$ & & & & \\
\hline$\Delta$ Agregate Earnings $(\%)$ & $\left(x_{i t}-x_{i t+(k-1)}\right) / x_{i t+(k-1)}$ & -0.0980 & & 0.1176 & & & \\
\hline GDP Growth & $g_{i t}$ & 0.0682 & & 0.0884 & 0.0100 & & \\
\hline GDP Growth (t-1) & $g_{i t+(k-1)}$ & 0.0551 & & 0.0998 & 0.0354 & 0.6300 & $* * *$ \\
\hline
\end{tabular}

Panel C: Developing Countries' Sample (n: 405)

\begin{tabular}{|c|c|c|c|c|c|c|c|c|}
\hline \multirow{2}{*}{\multicolumn{2}{|c|}{ Developing }} & \multirow{2}{*}{\multicolumn{2}{|c|}{$\frac{\text { Aggregate Earnings }}{X_{i t}}$}} & \multicolumn{2}{|c|}{$\Delta$ Agg. Earnings } & $\Delta$ Agg. Earnings $(\%)$ & \multicolumn{2}{|c|}{ GDP growth } \\
\hline & & & & $x_{i t}-x_{i t+}$ & $(k+1)$ & $\left(x_{i t}-x_{i t+(k-1)}\right) / x$ & $g_{i t}$ & \\
\hline$\Delta$ Agregate Earnings & $x_{i t}-x_{i t+(k-1)}$ & 0.3750 & $* * *$ & & & & & \\
\hline$\Delta$ Agregate Earnings $(\%)$ & $\left(x_{i t}-x_{i t+(k-1)}\right) / x_{i t+(k-1)}$ & 0.3900 & $* * *$ & 0.6540 & $* * *$ & & & \\
\hline GDP Growth & $g_{i t}$ & -0.0071 & & 0.0008 & & 0.0020 & & \\
\hline GDP Growth (t-1) & $g_{i t+(k-1)}$ & -0.0131 & & 0.0029 & & 0.0005 & 0.7800 & $* * *$ \\
\hline
\end{tabular}

Panel D: Asian Sample (n: 181)

\begin{tabular}{|c|c|c|c|c|c|c|c|}
\hline \multirow{2}{*}{\multicolumn{2}{|c|}{ Asia }} & \multirow{2}{*}{\multicolumn{2}{|c|}{$\frac{\text { Aggregate Earnings }}{X_{i t}}$}} & \multirow{2}{*}{$\frac{\Delta \text { Agg. Earnings }}{x_{i t}-x_{i t+(k+1)}}$} & \multirow{2}{*}{$\frac{\Delta \text { Agg. Earnings }(\%)}{\left(x_{i t}-x_{i t+(k-1)}\right) / x_{i t+(k-1)}}$} & \multirow{2}{*}{\multicolumn{2}{|c|}{$\begin{array}{c}\text { GDP growth } \\
g_{i t}\end{array}$}} \\
\hline & & & & & & & \\
\hline$\Delta$ Agregate Earnings & $x_{i t}-x_{i t+(k-1)}$ & 0.3240 & $* * *$ & & & & \\
\hline$\Delta$ Agregate Earnings $(\%)$ & $\left(x_{i t}-x_{i t+(k-1)}\right) / x_{i t+(k-1)}$ & -0.0887 & & 0.0076 & & & \\
\hline GDP Growth & $g_{i t}$ & 0.0142 & & 0.0134 & -0.0160 & & \\
\hline GDP Growth (t-1) & $g_{i t+(k-1)}$ & -0.0074 & & 0.0154 & -0.0050 & 0.8040 & $* * *$ \\
\hline
\end{tabular}

Panel E: African Sample (n: 224)

\begin{tabular}{|c|c|c|c|c|c|c|c|c|}
\hline \multirow{2}{*}{\multicolumn{2}{|c|}{ Africa }} & \multirow{2}{*}{\multicolumn{2}{|c|}{$\begin{array}{c}\text { Aggregate Earnings } \\
X_{i t} \\
\end{array}$}} & \multirow{2}{*}{\multicolumn{2}{|c|}{$\frac{\Delta \text { Agg. Earnings }}{x_{i t}-x_{i t+(k+1)}}$}} & \multirow{2}{*}{$\frac{\Delta \text { Agg. Earnings }(\%)}{\left(x_{i t}-x_{i t+(k-1)}\right) / x_{i t+(k-1)}}$} & \multicolumn{2}{|c|}{ GDP growth } \\
\hline & & & & & & & $g_{i t}$ & \\
\hline$\Delta$ Agregate Earnings & $x_{i t}-x_{i t+(k-1)}$ & 0.6970 & $* * *$ & & & & & \\
\hline$\Delta$ Agregate Earnings $(\%)$ & $\left(x_{i t}-x_{i t+(k-1)}\right) / x_{i t+(k-1)}$ & 0.9490 & $* * *$ & 0.6820 & $* * *$ & & & \\
\hline GDP Growth & $g_{i t}$ & -0.0097 & & 0.0025 & & -0.0039 & & \\
\hline GDP Growth (t-1) & $g_{i t+(k-1)}$ & -0.0073 & & 0.0010 & & -0.0022 & 0.5760 & $* * *$ \\
\hline
\end{tabular}

Panel F: Split Sample for the period of 1989-1996 (n: 104)

\begin{tabular}{|c|c|c|c|c|c|c|c|}
\hline \multirow{2}{*}{\multicolumn{2}{|c|}{ Period 1: 1989 - 1996}} & \multirow{2}{*}{\multicolumn{2}{|c|}{$\begin{array}{c}\text { Aggregate Earnings } \\
X_{i t} \\
\end{array}$}} & \multirow{2}{*}{$\frac{\Delta \text { Agg. Earnings }}{x_{i t}-x_{i t+(k+1)}}$} & \multirow{2}{*}{$\frac{\Delta \text { Agg. Earnings }(\%)}{\left(x_{i t}-x_{i t+(k-1)}\right) / x_{i t+(k-1)}}$} & \multirow{2}{*}{\multicolumn{2}{|c|}{$\frac{\text { GDP growth }}{g_{i t}}$}} \\
\hline & & & & & & & \\
\hline$\Delta$ Agregate Earnings & $x_{i t}-x_{i t+(k-1)}$ & 0.6670 & $* * *$ & & & & \\
\hline$\Delta$ Agregate Earnings $(\%)$ & $\left(x_{i t}-x_{i t+(k-1)}\right) / x_{i t+(k-1)}$ & -0.0777 & & 0.0351 & & & \\
\hline GDP Growth & $g_{i t}$ & 0.0286 & & 0.0759 & -0.0133 & & \\
\hline GDP Growth (t-1) & $g_{i t+(k-1)}$ & 0.0381 & & 0.0635 & 0.0063 & 0.7870 & $* * *$ \\
\hline
\end{tabular}

Panel G: Split Sample for the period of 1999-2007 (n: 265)

\begin{tabular}{|c|c|c|c|c|c|c|c|c|}
\hline \multirow{2}{*}{\multicolumn{2}{|c|}{ Period 2: 1999 - 2007}} & \multirow{2}{*}{\multicolumn{2}{|c|}{\begin{tabular}{|c} 
Aggregate Earnings \\
$X_{i t}$ \\
\end{tabular}}} & \multirow{2}{*}{\multicolumn{2}{|c|}{$\frac{\Delta \text { Agg. Earnings }}{x_{i t}-x_{i t+(k+1)}}$}} & \multirow{2}{*}{\begin{tabular}{|c|}
$\Delta$ Agg. Earnings $(\%)$ \\
$\left(x_{i t}-x_{i t+(k-1)}\right) / x_{i t+(k-1)}$
\end{tabular}} & \multirow{2}{*}{\multicolumn{2}{|c|}{$\begin{array}{c}\text { GDP growth } \\
g_{i t}\end{array}$}} \\
\hline & & & & & & & & \\
\hline$\Delta$ Agregate Earnings & $x_{i t}-x_{i t+(k-1)}$ & 0.5350 & $* * *$ & & & & & \\
\hline$\Delta$ Agregate Earnings $(\%)$ & $\left(x_{i t}-x_{i t+(k-1)}\right) / x_{i t+(k-1)}$ & 0.2130 & $* * *$ & 0.5630 & $* * *$ & & & \\
\hline GDP Growth & $g_{i t}$ & 0.0843 & & 0.0437 & & 0.0048 & & \\
\hline GDP Growth (t-1) & $g_{i t+(k-1)}$ & 0.0673 & & 0.0494 & & 0.0027 & 0.7260 & $* * *$ \\
\hline
\end{tabular}


Panel H: Split Sample for the period of 2010-2015 (n: 215)

\begin{tabular}{|c|c|c|c|c|c|c|c|}
\hline \multirow{2}{*}{\multicolumn{2}{|c|}{ Period 3: 2010 - 2015}} & \multirow{2}{*}{\multicolumn{2}{|c|}{\begin{tabular}{|c} 
Aggregate Earnings \\
$X_{i t}$
\end{tabular}}} & \multirow{2}{*}{$\Delta$ Agg. Earnings } & \multirow{2}{*}{$\Delta$ Agg. Earnings $(\%)$} & \multirow{2}{*}{\multicolumn{2}{|c|}{$\begin{array}{c}\text { GDP growth } \\
g_{i t}\end{array}$}} \\
\hline & & & & & & & \\
\hline$\Delta$ Agregate Earnings & $x_{i t}-x_{i t+(k-1)}$ & 0.4950 & *** & & & & \\
\hline$\Delta$ Agregate Earnings $(\%)$ & $\left(x_{i t}-x_{i t+(k-1)}\right) / x_{i t+(k-1)}$ & -0.0378 & & 0.0326 & & & \\
\hline GDP Growth & $g_{i t}$ & -0.0599 & & -0.0318 & -0.0002 & & \\
\hline GDP Growth (t-1) & $g_{i t+(k-1)}$ & -0.1079 & & -0.0265 & 0.0186 & -0.1110 & \\
\hline
\end{tabular}

Note: ${ }^{* * *}, * *$, and $*$ significant at $1.00 \%, 5.00 \%$, and $10.00 \%$ significance levels respectively.

\subsection{Analysis}

The analysis shows that the aggregate of accounting earnings does not associate with GDP growth. Table 3 shows that the aggregate of the accounting earnings $\left(\mathrm{X}_{\mathrm{it}}\right)$ does not associate with the beta value of 0.000 and $\mathrm{t}$-value of 0.5146 in Model 1. Thus, hypothesis $\mathrm{H}_{\mathrm{A} 1}$ is not supported. It depicts that the aggregate of accounting earnings cannot explain or predict next year's GDP. The same test undertaken for the changes in the aggregate of the accounting earnings $\left(\Delta \mathrm{X}_{\mathrm{it}}\right)$ produces a beta value of 0.0000 and a $\mathrm{t}$ value of 0.7108 in Model 2. It shows that hypothesis $\mathrm{H}_{\mathrm{A} 2}$ is not supported, meaning that changes in the aggregate of accounting earnings do not associate statistically. This study proposes a methodology refinement concept by changing the aggregate of accounting earnings into the changes in earnings aggregate. It also shows that this methodological refinement is not supported.

Model 3 includes the GDP growth predictor numbers for the previous year $\left(\mathrm{g}_{\mathrm{it}+}(\mathrm{k}-1)\right.$. A regression test shows that those numbers are significantly associated with a beta value of 0.7742 and a $t$-value of 24.9425 . Those numbers are standard for GDP growth predictor numbers for the next year, as stated in the economic literature. On the other hand, this study undertakes a retest in Model 4 by changing the

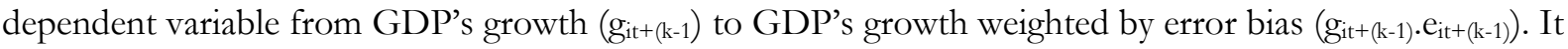
shows that the previous year's GDP growth predictor is associated with next year's GDP growth, as evidenced by t-values of 22.4349 in Model 4 and 11.0146 in Model 5. It means that GDP, and its modified growth as control variables, associate statistically and significantly against future GDP growth. Nonetheless, changes in the aggregate of the accounting earnings cannot predict next year's GDP growth, with the t-value of 0.7232 . Therefore, hypotheses $\mathrm{H}_{\mathrm{A} 2}$ is not supported. This examination of the

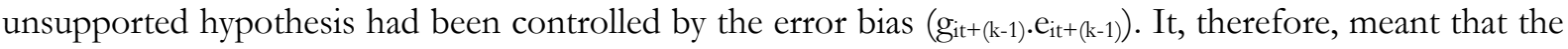
unsupported hypothesis has validity.

Initially, this study separates the samples into Asian and African countries. The analysis shows that the aggregate of accounting earnings is not able to predict next year's GDP, either for Asia-Pacific or African countries. The t-value in Model 1 is 0.1898 and -0.1439 for Asia-Pacific and Africa, respectively. After the partition, hypotheses $\mathrm{H}_{\mathrm{A} 1}$ is, again, not supported. Similarly, changes to the aggregate of accounting earnings in Model 2 produce the t-values of 0.1794 and 0.0367 for Asia-Pacific and African countries, respectively. It depicts that refinements to the changes in the numbers are not supported, and hypothesis $\mathrm{H}_{\mathrm{A} 2}$ is not supported either. Nevertheless, the previous year's GDP growth predictor is consistently able to predict next year's GDP growth. However, hypotheses $\mathrm{H}_{\mathrm{A} 1}$ and $\mathrm{H}_{\mathrm{A} 2}$ are not supported in Models 3, 4, and 5. 
Table 3

Main regression tests results

\begin{tabular}{|c|c|c|c|c|c|c|c|c|c|c|c|c|c|c|c|c|c|}
\hline \multirow{10}{*}{ 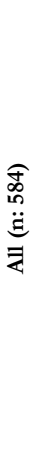 } & \multirow{2}{*}{$\operatorname{Var}(s)$. } & \multicolumn{4}{|c|}{ Model 1} & \multicolumn{3}{|c|}{ Model 2} & \multicolumn{3}{|c|}{ Model 3} & \multicolumn{3}{|c|}{ Model 4} & \multicolumn{3}{|c|}{ Model 5} \\
\hline & & Pred. & $\beta$ & $\mathbf{t}$ & Sig. & $\beta$ & $\mathbf{t}$ & Sig. & $\beta$ & $\mathrm{t}$ & Sig. & $\beta$ & $\mathbf{t}$ & Sig. & $\beta$ & $\mathbf{t}$ & Sig. \\
\hline & $a_{k}$ & & 0.0543 & 0.2548 & & 0.0682 & 0.3389 & & 0.0577 & 0.4118 & & 0.3160 & 2.1362 & ** & 0.1751 & 1.2943 & \\
\hline & $X_{i t}$ & + & 0.0000 & 0.5146 & & & & & & & & & & & & & \\
\hline & $\Delta X_{i t}$ & + & & & & 0.0000 & 0.7108 & & 0.0000 & 0.1481 & & 0.0000 & 0.7232 & & 0.0000 & 0.3354 & \\
\hline & $g_{i t+(k+1)}$ & + & & & & & & & 0.7742 & 24.9425 & $* * *$ & & & & 0.5145 & 11.0146 & $* * *$ \\
\hline & 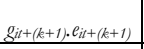 & + & & & & & & & & & & 0.0503 & 22.4349 & $* * *$ & 0.0231 & 7.2158 & $* * *$ \\
\hline & F V alue & & & 0.2648 & & & 0.5053 & & & 311.5861 & $* * *$ & & 252.1320 & *** & & 243.3377 & *** \\
\hline & $R^{2}$ & & $0.05 \%$ & & & $0.09 \%$ & & & $51.75 \%$ & & & $46.46 \%$ & & & $55.73 \%$ & & \\
\hline & Adj-R $\mathrm{R}^{2}$ & & $-0.13 \%$ & & & $-0.08 \%$ & & & $51.58 \%$ & & & $46.28 \%$ & & & $55.50 \%$ & & \\
\hline
\end{tabular}

\begin{tabular}{|c|c|c|c|c|c|c|c|c|c|c|c|c|c|c|c|c|c|}
\hline \multirow{10}{*}{ 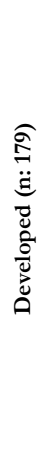 } & \multirow{2}{*}{$\operatorname{Var}(\mathrm{s})$. } & \multicolumn{4}{|c|}{ Model 1} & \multicolumn{3}{|c|}{ Model 2} & \multicolumn{3}{|c|}{ Model 3} & \multicolumn{3}{|c|}{ Model 4} & \multicolumn{3}{|c|}{ Model 5} \\
\hline & & Pred. & $\beta$ & $\mathrm{t}$ & Sig. & $\beta$ & $\mathrm{t}$ & Sig. & $\beta$ & $\mathrm{t}$ & Sig. & $\beta$ & $\mathrm{t}$ & Sig. & $\beta$ & $\mathrm{t}$ & Sig. \\
\hline & $a_{k}$ & & -0.3482 & -0.7405 & & -0.3104 & -0.7315 & & -0.0122 & -0.0368 & & -0.0126 & -0.0372 & & 0.0624 & 0.2015 & \\
\hline & $X_{i t}$ & + & 0.0000 & 0.9093 & & & & & & & & & & & & & \\
\hline & $\Delta X_{i t}$ & + & & & & 0.0000 & 1.1811 & & 0.0000 & 0.4391 & & 0.0000 & 1.3071 & & 0.0000 & 0.7532 & \\
\hline & $g_{i+1+(k+1)}$ & + & & & & & & & 0.6222 & 10.6698 & $* * *$ & & & & 0.4064 & 6.0083 & *** \\
\hline & $g_{i t+(k+1)} \cdot e_{i t+(k+1}$ & + & & & & & & & & & & 0.0412 & 10.1714 & *** & 0.0246 & 5.3418 & *** \\
\hline & F V alue & & & 0.8269 & & & 1.3951 & & & 58.0649 & *** & & 52.8302 & $* * *$ & & 54.2776 & *** \\
\hline & $R^{2}$ & & $0.47 \%$ & & & $0.78 \%$ & & & $39.75 \%$ & & & $37.51 \%$ & & & $48.20 \%$ & & \\
\hline & $A d j-\mathrm{R}^{2}$ & & $-0.10 \%$ & & & $0.22 \%$ & & & $39.07 \%$ & & & $36.80 \%$ & & & $47.31 \%$ & & \\
\hline
\end{tabular}

\begin{tabular}{|c|c|c|c|c|c|c|c|c|c|c|c|c|c|c|c|c|c|}
\hline \multirow{10}{*}{ 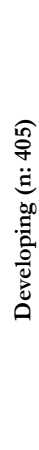 } & \multirow{2}{*}{$\operatorname{Var}(\mathrm{s})$. } & \multicolumn{4}{|c|}{ Model 1} & \multicolumn{3}{|c|}{ Model 2} & \multicolumn{3}{|c|}{ Model 3} & \multicolumn{3}{|c|}{ Model 4} & \multicolumn{3}{|c|}{ Model 5} \\
\hline & & Pred. & $\beta$ & $\mathrm{t}$ & Sig. & $\beta$ & $\mathrm{t}$ & Sig. & $\beta$ & $\mathrm{t}$ & Sig. & $\beta$ & $\mathrm{t}$ & Sig. & $\beta$ & $\mathrm{t}$ & Sig. \\
\hline & $a_{k}$ & & 0.1958 & 0.8129 & & 0.1846 & 0.8084 & & 0.0324 & 0.2263 & & 0.3566 & 2.0954 & ** & 0.1097 & 0.7736 & \\
\hline & $X_{i t}$ & + & 0.0000 & -0.1427 & & & & & & & & & & & & & \\
\hline & $\Delta X_{i t}$ & + & & & & 0.0000 & 0.0156 & & 0.0000 & -0.0474 & & 0.0000 & -0.0118 & & 0.0000 & -0.0460 & \\
\hline & $g_{i+(k+1)}$ & + & & & & & & & 0.8859 & 24.9572 & $* * *$ & & & & 0.7226 & 13.7032 & $* * *$ \\
\hline & $g_{i t+(k+1)} \cdot e_{i t+(k+1)}$ & + & & & & & & & & & & 0.0550 & 18.0442 & *** & 0.0157 & 4.1228 & $* * *$ \\
\hline & F V alue & & & 0.0204 & & & 0.0002 & & & 311.4321 & *** & & 162.7962 & *** & & 221.5495 & $* * *$ \\
\hline & $R^{2}$ & & $0.01 \%$ & & & $0.00 \%$ & & & $60.78 \%$ & & & $44.75 \%$ & & & $62.37 \%$ & & \\
\hline & $A d j-R^{2}$ & & $-0.24 \%$ & & & $-0.25 \%$ & & & $60.58 \%$ & & & $44.47 \%$ & & & $62.09 \%$ & & \\
\hline
\end{tabular}

\begin{tabular}{|c|c|c|c|c|c|c|c|c|c|c|c|c|c|c|c|c|c|}
\hline \multirow{10}{*}{ 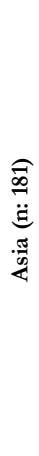 } & \multirow{2}{*}{$\operatorname{Var}(\mathrm{s})}$. & \multicolumn{4}{|c|}{ Model 1} & \multicolumn{3}{|c|}{ Model 2} & \multicolumn{3}{|c|}{ Model 3} & \multicolumn{3}{|c|}{ Model 4} & \multicolumn{3}{|c|}{ Model 5} \\
\hline & & Pred. & $\beta$ & $t$ & Sig. & $\beta$ & $t$ & Sig. & $\beta$ & $t$ & Sig. & $\beta$ & $t$ & Sig. & $\beta$ & $t$ & Sig. \\
\hline & $a_{k}$ & & -0.1699 & -0.3131 & & -0.1472 & -0.2934 & & -0.1936 & -0.6470 & & 0.3010 & 0.8112 & & -0.0924 & -0.3075 & \\
\hline & $X_{i t}$ & + & 0.0000 & 0.1898 & & & & & & & & & & & & & \\
\hline & $\Delta X_{i t}$ & + & & & & 0.0000 & 0.1794 & & 0.0000 & 0.0234 & & 0.0000 & -0.0432 & & 0.0000 & -0.0133 & \\
\hline & $g_{i+1(k+1)}$ & + & & & & & & & 0.9102 & 18.0177 & *** & & & & 0.7846 & 9.9479 & $* * *$ \\
\hline & $g_{i+1}(k+1) \cdot e_{i+1}+(k+1$ & + & & & & & & & & & & 0.0515 & 12.3342 & $* * *$ & 0.0109 & 2.0619 & ** \\
\hline & F V alue & & & 0.0360 & & & 0.0322 & & & 162.3639 & $* * *$ & & 76.0962 & $* * *$ & & 111.6368 & *** \\
\hline & $R^{2}$ & & $0.02 \%$ & & & $0.02 \%$ & & & $64.59 \%$ & & & $46.09 \%$ & & & $65.42 \%$ & & \\
\hline & $A d j-R^{2}$ & & $-0.54 \%$ & & & $-0.54 \%$ & & & $64.20 \%$ & & & $45.49 \%$ & & & $64.84 \%$ & & \\
\hline
\end{tabular}




\begin{tabular}{|c|c|c|c|c|c|c|c|c|c|c|c|c|c|c|c|c|c|}
\hline \multirow{10}{*}{ 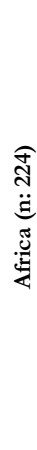 } & \multirow{2}{*}{$\operatorname{Var}(\mathrm{s})$} & \multicolumn{4}{|c|}{ Model 1} & \multicolumn{3}{|c|}{ Model 2} & \multicolumn{3}{|c|}{ Model 3} & \multicolumn{3}{|c|}{ Model 4} & \multicolumn{3}{|c|}{ Model 5} \\
\hline & & Pred. & $\beta$ & $\mathrm{t}$ & Sig. & $\beta$ & $\mathrm{t}$ & Sig. & $\beta$ & $\mathrm{t}$ & Sig. & $\beta$ & $\mathrm{t}$ & Sig. & $\beta$ & $\mathrm{t}$ & Sig. \\
\hline & $a_{k}$ & & 0.4361 & 3.2106 & **** & 0.4333 & 3.2233 & $* * *$ & 0.2671 & 2.4011 & ** & 0.3054 & 2.4908 & ** & 0.2787 & 2.5312 & ** \\
\hline & $X_{i t}$ & + & 0.0000 & -0.1439 & & & & & & & & & & & & & \\
\hline & $\Delta X_{i t}$ & + & & & & 0.0000 & 0.0367 & & 0.0000 & 0.0339 & & 0.0000 & 0.0315 & & 0.0000 & 0.0361 & \\
\hline & $g_{i+1}(k+1)$ & + & & & & & & & 0.6638 & 10.4842 & $* * *$ & & & & 0.9323 & 7.3672 & $* * *$ \\
\hline & $g_{i t+(k+1)} \cdot e_{i t+(k+1)}$ & + & & & & & & & & & & 0.1308 & 7.1853 & *** & -0.0806 & -2.4418 & \\
\hline & F V alue & & & 0.0207 & & & 0.0013 & & & 54.9604 & $* * *$ & & 25.8151 & $* * *$ & & 39.4505 & **** \\
\hline & $R^{2}$ & & $0.01 \%$ & & & $0.00 \%$ & & & $33.22 \%$ & & & $18.94 \%$ & & & $34.98 \%$ & & \\
\hline & $A d j-R^{2}$ & & $-0.44 \%$ & & & $-0.45 \%$ & & & $32.61 \%$ & & & $18.20 \%$ & & & $34.09 \%$ & & \\
\hline
\end{tabular}

Note: $* * *, * *$, and $*$ significant at $1.00 \%, 5.00 \%$, and $10.00 \%$ significance levels respectively. $X_{i t}$ is the aggregate of accounting earnings; $\Delta X_{i t}=X_{i t}-X_{i t-1}$ is the aggregate of accounting earnings' growth; $g_{i t+1}$ is next year's GDP growth; for the Model 4 and 5 , the independent variable is transformed from $g_{i t+1}$ into $g_{i t+1 .} e_{i t}$ as growth modified process; $(\mathrm{k}+\mathrm{n}), \mathrm{n}=1$ and 2 show lead and lag year $\mathrm{n}$.

The result of the test inducing the previous year's GDP growth predictor can improve the fitness of the model. The model's fitness increases in its Adj- $\mathrm{R}^{2}$ from Model 1 to Model 2 valued at $-0.13 \%$ and $0.08 \%$, respectively, relative to Model 3, Model 4 and Model 5 valued at 51.58\%, 46.28\%, and 55.50\%, respectively, for all the samples. The result of the sample split into Asia-Pacific, and African countries also show the equivalence of the model's level of fitness, as depicted in the sample as a whole.

\subsection{Analysis}

This study undertook a periodical sensitivity test. Apart from the partition test shown in Table 3, this study can only undertake a periodical basis sensitivity test. The reason is that a country basis partition cannot be done as the research period excludes the periods between 1997-1998 and 2008-2009. Likewise, most developing countries do not have adequate data and information related to their GDPs' growth, as well as fluctuating aggregates of their accounting earnings data that could not be presented logically. The result of the sensitivity test is presented in Table 4.

Table 4

Regression sensitivity - periodically sensitivity test results

\begin{tabular}{|c|c|c|c|c|c|c|c|c|c|c|c|c|c|c|c|c|c|}
\hline \multirow{10}{*}{ 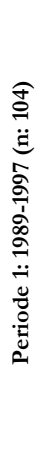 } & \multirow{2}{*}{$\operatorname{Var}(\mathrm{s})}$. & \multicolumn{4}{|c|}{ Model 1} & \multicolumn{3}{|c|}{ Model 2} & \multicolumn{3}{|c|}{ Model 3} & \multicolumn{3}{|c|}{ Model 4} & \multicolumn{3}{|c|}{ Model 5} \\
\hline & & Pred. & $\beta$ & $t$ & Sig. & $\beta$ & $t$ & Sig. & $\beta$ & $t$ & Sig. & $\beta$ & $\mathrm{t}$ & Sig. & $\beta$ & $\mathrm{t}$ & Sig. \\
\hline & $a_{k}$ & & 0.8536 & 2.4055 & ** & 0.8268 & 2.5268 & ** & 0.2729 & 1.3126 & & 0.6306 & 2.1713 & $* *$ & 0.2690 & 1.2912 & \\
\hline & $X_{i t}$ & + & 0.0000 & 0.2887 & & & & & & & & & & & & & \\
\hline & $\Delta X_{i t}$ & + & & & & 0.0000 & 0.7689 & & 0.0000 & 0.4231 & & 0.0000 & 0.9202 & & 0.0000 & 0.3661 & \\
\hline & $g_{i+1+(k+1)}$ & + & & & & & & & 1.0109 & 12.7705 & $* * *$ & & & & 1.0685 & 10.1133 & $* * *$ \\
\hline & $g_{i t+(k+1) \cdot e_{i+(k+1)}}$ & + & & & & & & & & & & 0.2680 & 5.5176 & $* * *$ & -0.0377 & -0.8247 & \\
\hline & F V alue & & & 0.0834 & & & 0.5912 & & & 82.3086 & $* * *$ & & 15.6028 & $* * *$ & & 54.9253 & $* * *$ \\
\hline & $R^{2}$ & & $0.08 \%$ & & & $0.58 \%$ & & & $61.98 \%$ & & & $23.60 \%$ & & & $62.23 \%$ & & \\
\hline & $A d j-\mathrm{R}^{2}$ & & $-0.90 \%$ & & & $-0.40 \%$ & & & $61.22 \%$ & & & $22.09 \%$ & & & $61.10 \%$ & & \\
\hline
\end{tabular}




\begin{tabular}{|c|c|c|c|c|c|c|c|c|c|c|c|c|c|c|c|c|c|}
\hline \multirow{10}{*}{ 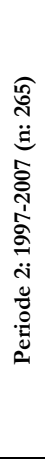 } & \multirow{2}{*}{$\operatorname{Var}(\mathrm{s})$. } & \multicolumn{4}{|c|}{ Model 1} & \multicolumn{3}{|c|}{ Model 2} & \multicolumn{3}{|c|}{ Model 3} & \multicolumn{3}{|c|}{ Model 4} & \multicolumn{3}{|c|}{ Model 5} \\
\hline & & Pred. & $\beta$ & $\mathbf{t}$ & Sig. & $\beta$ & $\mathbf{t}$ & Sig. & $\beta$ & $\mathbf{t}$ & Sig. & $\beta$ & $\mathbf{t}$ & Sig. & $\beta$ & $\mathbf{t}$ & Sig. \\
\hline & $a_{k}$ & & -0.3191 & -0.7142 & & -0.1413 & -0.3350 & & -0.0819 & -0.2817 & & 0.4234 & 1.4034 & & 0.1776 & 0.6330 & \\
\hline & $X_{i t}$ & + & 0.0000 & 1.3712 & & & & & & & & & & & & & \\
\hline & $\Delta X_{i t}$ & + & & & & 0.0000 & 0.7087 & & 0.0000 & 0.1839 & & 0.0000 & 0.7159 & & 0.0000 & 0.3796 & \\
\hline & $g_{i t+(k+1)}$ & + & & & & & & & 0.7710 & 17.0577 & $* * *$ & & & & 0.4765 & 6.8500 & $* * *$ \\
\hline & $g_{i+(k+1) \cdot, e_{i+1}+(k+1)}$ & + & & & & & & & & & & 0.0498 & 16.0849 & *** & 0.0249 & 5.3823 & $* * *$ \\
\hline & F V alue & & & 1.8803 & & & 0.5022 & & & 146.0102 & *** & & 129.8591 & *** & & 117.3878 & *** \\
\hline & $R^{2}$ & & $0.71 \%$ & & & $0.19 \%$ & & & $52.71 \%$ & & & $49.78 \%$ & & & $57.43 \%$ & & \\
\hline & Adj $-\mathrm{R}^{2}$ & & $0.33 \%$ & & & $-0.19 \%$ & & & $52.35 \%$ & & & $49.40 \%$ & & & $56.94 \%$ & & \\
\hline
\end{tabular}

\begin{tabular}{|c|c|c|c|c|c|c|c|c|c|c|c|c|c|c|c|c|c|}
\hline \multirow{10}{*}{ 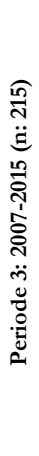 } & \multirow{2}{*}{$\operatorname{Var}(\mathbf{s})}$. & \multicolumn{4}{|c|}{ Model 1} & \multicolumn{3}{|c|}{ Model 2} & \multicolumn{3}{|c|}{ Model 3} & \multicolumn{3}{|c|}{ Model 4} & \multicolumn{3}{|c|}{ Model 5} \\
\hline & & Pred. & $\beta$ & $t$ & Sig. & $\beta$ & $\mathrm{t}$ & Sig. & $\beta$ & $\mathrm{t}$ & Sig. & $\beta$ & $t$ & Sig. & $\beta$ & $\mathrm{t}$ & Sig. \\
\hline & $a_{k}$ & & -0.0432 & -0.6661 & & -0.0624 & -1.0456 & & -0.0777 & -1.2914 & & -0.0745 & -1.3002 & & -0.0487 & -0.8464 & \\
\hline & $X_{i t}$ & + & 0.0000 & -0.8752 & & & & & & & & & & & & & \\
\hline & $\Delta X_{i t}$ & + & & & & 0.0000 & -0.4640 & & 0.0000 & -0.5092 & & 0.0000 & -0.5294 & & 0.0000 & -0.4650 & \\
\hline & $g_{i+1+(k+1)}$ & + & & & & & & & -0.1095 & -1.6405 & & & & & 0.2401 & 2.4840 & $* *$ \\
\hline & $g_{i t+(k+1)}, e_{i+1(k+1)}$ & + & & & & & & & & & & -0.1434 & -4.3979 & & -0.2352 & -4.7972 & \\
\hline & F Value & & & 0.7661 & & & 0.2153 & & & 1.4541 & & & 9.7877 & $* * *$ & & 8.7410 & $* * *$ \\
\hline & $R^{2}$ & & $0.36 \%$ & & & $0.10 \%$ & & & $1.35 \%$ & & & $8.45 \%$ & & & $11.05 \%$ & & \\
\hline & $A d j-R^{2}$ & & $-0.11 \%$ & & & $-0.37 \%$ & & & $0.42 \%$ & & & $7.59 \%$ & & & $9.79 \%$ & & \\
\hline
\end{tabular}

Note: $* * *, * *$, and $*$ significant at $1.00 \%, 5.00 \%$, and $10.00 \%$ significance levels respectively. $X_{i t}$ is the aggregate of accounting earnings; $\Delta X_{i t}=X_{i t}-X_{i t-1}$ is the aggregate of accounting earnings' growth; $g_{i t+1}$ is next year's GDP growth; for Models 4 and 5 , the independent variable is transformed from $g_{i t+1}$ into $g_{i t+1 \cdot i t}$ as a growth modified process; $(\mathrm{k}+\mathrm{n}), \mathrm{n}=1$ and 2 show lead and lag year $\mathrm{n}$.

Table 4 depicts that the aggregate of the accounting earnings is not associated with next year's GDP growth, having a beta value of 0.0000 and the $t$-value of 0.2887 in period 1 , meaning that hypothesis $\mathrm{H}_{\mathrm{A} 1}$ is not supported. It shows that the aggregate of accounting earnings is not a good predictor of future growth in GDP. Nevertheless, this study provides some evidence that changes in the aggregate of the accounting earnings cannot predict next year's GDP growth, which can be seen in Model 2. Therefore, hypothesis $\mathrm{H}_{\mathrm{A} 2}$ is not supported. Furthermore, changes in the aggregate of the accounting earnings cannot predict next year's GDP growth in Models 3, 4, and 5. Thus, this also supports that the proposed methodology refinement is supported.

This study presents supporting evidence of hypothesis $\mathrm{H}_{\mathrm{A} 2}$ in period-2 and -3 . The sample partition for period-2 and -3 cannot provide empirical evidence to support hypotheses $\mathrm{H}_{\mathrm{A} 1}$ and $\mathrm{H}_{\mathrm{A} 2}$. However, the previous year's GDP growth predictor and its modified version are still consistent as predictor numbers for next year's GDP growth for all periods. For example, in period 2, GDP's growth predictor has a beta value of 0.4765 and 0.0249 for Model 5 , with a significance level of $1.00 \%$.

The periodical sample partition depicts the improvement in the model's fitness, as shown in the previous test. This improvement is a result of the inducement of the previous year's GDP growth predictor. The level of the model's improvement can be seen from the increase in the Adj- $\mathrm{R}^{2}$ from Model 1 and Model 2, having values of minus $0.90 \%$ and $0.40 \%$ respectively, relative to Models 3, 4 and 5, which have Adj-R2 values of $61.22 \%, 22.09 \%$, and $61.10 \%$ respectively, for the data period 1 . 


\subsection{Selected sample: Developed countries and positive earnings growth}

This study cannot find evidence that accounting earnings are used to predict next year's GDP growth. Therefore, this study undertakes a one-sided test using a selected sample. The sample is from the developed countries whose earnings growth is positive. The selected sample is Australia, China, Hong Kong, Japan, New Zealand, Singapore, South Korea, and Taiwan during the period from 1989-2015. The results are presented in Table 5.

In the selected sample, the aggregate of accounting earnings is positively associated with next year's GDP growth, producing the $\mathrm{t}$-value of 1.9667 at 10\% significance level in Model 2. Thus, hypothesis $\mathrm{H}_{\mathrm{A} 2}$ is supported. It explains that the aggregate of the accounting earnings can predict next year's GDP growth. After including the previous year's GDP growth and its modified predictors, changes in the aggregate of the accounting earnings are positively influenced, amounting to 0.0000 with the $t$-value of 1.8727 at $10 \%$ significance level in Model 3. Thus, hypothesis $\mathrm{H}_{\mathrm{A} 2}$ is supported. The statistical result is consistent with Model 4. This study shows that changes in the aggregate of the accounting earnings have a role as the next year's GDP growth predictor, but only for those developed countries whose earnings growth is positive. However, the aggregate of accounting earnings cannot predict the next year's GDP growth for developing countries whose earnings growth is also positive.

Table 5

Developed countries regression results

\begin{tabular}{|c|c|c|c|c|c|c|c|c|c|c|c|c|c|c|c|c|c|}
\hline \multirow{10}{*}{ 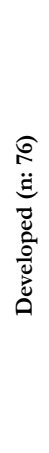 } & \multirow{2}{*}{$\operatorname{Var}(\mathrm{s})$. } & \multicolumn{4}{|c|}{ Model 1} & \multicolumn{3}{|c|}{ Model 2} & \multicolumn{3}{|c|}{ Model 3} & \multicolumn{3}{|c|}{ Model 4} & \multicolumn{3}{|c|}{ Model 5} \\
\hline & & Pred. & $\beta$ & $t$ & Sig. & $\beta$ & $\mathrm{t}$ & Sig. & $\beta$ & $\mathrm{t}$ & Sig. & $\beta$ & $\mathrm{t}$ & Sig. & $\beta$ & $\mathrm{t}$ & Sig. \\
\hline & $a_{k}$ & & 1.4202 & 4.0695 & *** & 1.3537 & 4.1730 & *** & 1.2462 & 4.3896 & $* * *$ & 1.3604 & 4.4442 & $* * *$ & 1.0743 & 3.9198 & **** \\
\hline & $X_{i t}$ & + & 0.0000 & 1.3209 & & & & & & & & & & & & & \\
\hline & $\Delta X_{i t}$ & + & & & & 0.0000 & 1.9667 & * & 0.0000 & 1.8727 & * & 0.0000 & 1.9928 & $*$ & 0.0000 & 1.6258 & \\
\hline & $g_{i+1(k+1)}$ & + & & & & & & & 0.2533 & 4.9188 & $* * *$ & & & & 0.6229 & 4.8463 & $* * *$ \\
\hline & $g_{i+1(k+1)}, e_{i+1}(k+1)$ & + & & & & & & & & & & 0.0314 & 3.1803 & $* * *$ & -0.0708 & -3.1070 & \\
\hline & F V alue & & & 1.7448 & & & 3.8679 & $*$ & & 14.6375 & **** & & 7.2292 & **** & & 14.1330 & *** \\
\hline & $R^{2}$ & & $2.30 \%$ & & & $4.97 \%$ & & & $28.62 \%$ & & & $16.53 \%$ & & & $37.06 \%$ & & \\
\hline & $A d j-R^{2}$ & & $0.98 \%$ & & & $3.68 \%$ & & & $26.67 \%$ & & & $14.24 \%$ & & & $34.44 \%$ & & \\
\hline
\end{tabular}

\begin{tabular}{|c|c|c|c|c|c|c|c|c|c|c|c|c|c|c|c|c|c|}
\hline \multirow{10}{*}{ 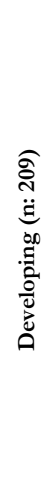 } & \multirow{2}{*}{$\operatorname{Var}(\mathrm{s})}$. & \multicolumn{4}{|c|}{ Model 1} & \multicolumn{3}{|c|}{ Model 2} & \multicolumn{3}{|c|}{ Model 3} & \multicolumn{3}{|c|}{ Model 4} & \multicolumn{3}{|c|}{ Model 5} \\
\hline & & Pred. & $\beta$ & t & Sig. & $\beta$ & t & Sig. & $\beta$ & $t$ & Sig. & $\beta$ & $t$ & Sig. & $\beta$ & t & Sig. \\
\hline & $a_{k}$ & & 1.6723 & 5.8318 & **** & 1.5548 & 5.6814 & *** & 0.7314 & 5.7561 & $* * *$ & 0.9989 & 6.3530 & $* * *$ & 0.6696 & 5.2861 & *** \\
\hline & $X_{i t}$ & + & 0.0000 & -1.3180 & & & & & & & & & & & & & \\
\hline & $\Delta X_{i t}$ & + & & & & 0.0000 & -0.0785 & & 0.0000 & $\begin{array}{c}-0.0876 \\
\end{array}$ & & 0.0000 & $\begin{array}{c}-0.0406 \\
\end{array}$ & & 0.0000 & $\begin{array}{c}-0.1064 \\
\end{array}$ & \\
\hline & $g_{i t+(k+1)}$ & + & & & & & & & 0.8374 & 28.4045 & $* * *$ & & & & 1.1052 & 11.3927 & *** \\
\hline & $g_{i t+(k+1)} \cdot e_{i+1}+(k+1)$ & + & & & & & & & & & & 0.1945 & 20.9637 & **** & -0.0705 & -2.8927 & \\
\hline & \begin{tabular}{|l|l} 
F Value \\
\end{tabular} & & & 1.7371 & & & 0.0062 & & & 403.4224 & $* * *$ & & 219.7476 & **** & & 281.3564 & **** \\
\hline & $R^{2}$ & & $0.83 \%$ & & & $0.00 \%$ & & & $79.66 \%$ & & & $68.09 \%$ & & & $80.46 \%$ & & \\
\hline & $A d j-R^{2}$ & & $0.35 \%$ & & & $-0.48 \%$ & & & $79.46 \%$ & & & $67.78 \%$ & & & $80.17 \%$ & & \\
\hline
\end{tabular}

Note: $* * * * *$, and $*$ significant at $1.00 \%, 5.00 \%$, and $10.00 \%$ significance levels respectively. $X_{i t}$ is the aggregate of accounting earnings; $\Delta X_{i t}=X_{i t}-X_{i t-1}$ is the aggregate of accounting earnings' growth; $g_{i t+1}$ is next year's GDP growth; for the Models 4 and 5, the independent variable is transformed from $g_{i+1}$ into $g_{i t+1} \cdot e_{i t}$ as a growth modified process; $(\mathrm{k}+\mathrm{n}), \mathrm{n}=1$ and 2 show lead and lag year $\mathrm{n}$. 
This study examines the country-group as well as the periodic basis for developing countries. However, aggregate earnings and their changes cannot predict future GDP growth. Therefore, this study does not present it. On the other hand, the degree of fitness of the model for developed countries shows significant results. The model's fitness can be shown by the high Adj- $\mathrm{R}^{2}$ value amounting to $26.67 \%$ in Model 3, increasing to $34.44 \%$ in Model 5. Therefore, this study can support the methodology refinement concept in which changes in the aggregate of the accounting earnings acts as next year's GDP growth predictor in developing countries.

\section{DISCUSSION AND FINDINGS}

This study finds that the aggregate of accounting earnings is not able to predict next year's GDP growth for developing countries. Similarly, changes in the aggregate of accounting earnings are not associated with GDP growth. Although Gonedes (1973b), Gonedes (1973a), and Brown and Ball (1967) conclude that accounting earnings have an immense influence on national economies, this study does not support this concept.

Konchitchki and Patatoukas (2014) and Patatoukas (2014) argue that the aggregate of accounting earnings influences national macro economies. They also depict that the aggregate of accounting earnings has an informativeness level, not only at the firm level but also at the market-wide and nation-wide levels. These studies conclude that accounting earnings do have properties. This concept is true. However, it does not yet consider the position of those accounting earnings' properties. It means that those properties are real when in the context of the developed countries whose earnings growth is positive, but not in the context of the developing countries.

Theories and concepts generally state that GDP measures the income from all the entities and households involved in any economic activities within a country (Becker et al., 2005; Fleurbaey, 2009; Fleurbaey \& Gaulier, 2009; Henderson et al., 2012). In other words, GDP primarily measures the performance of a country's economic activities. The results of this study do not reject the concepts proposed by these studies. Nevertheless, this mirroring concept has to consider the administrative and accounting capturing process. Konchitchki and Patatoukas (2014), McCloskey (1993), and Konchitchki (2011) argue that the total numbers of the accounting earnings of firms reflected by the GDP's growth from year to year should be noted when those total numbers are calculated correctly and comprehensively.

Fama (1981), Fischer and Merton (1984), Harvey (1989), and Shevlin (1990) argue that economic growth is the denominator of the capital and bond markets' developments. These studies argue that a national income is the sum of the progressive firms' taxes from all of the firms' incomes and profits, and conversely, for all of the firms' expenses and losses within a country. All these studies argue that there is conformity between the aggregate of the accounting earnings, capital markets, and GDP's movement. However, they researched a country in which the capital market is relatively close to being strong (according to the capital market hypothesis). This hypothesis argues that national resources allocations are efficient. In the case of developing countries, productive resources' allocations might not occur since their economies are still fractional or only partially developed.

According to the previous discussions, this study finds that the aggregate of accounting earnings has the same properties in every capital market within a country. Nevertheless, those properties should be understood conditionally and contingently, especially in developing countries, relative to developed countries. The differences in accounting earnings properties and the inability of the aggregate of accounting earnings to predict the next year's GDP are caused by some factors as follows:

Firstly, developing countries have more considerable future economic uncertainties than in developed countries. Production factors in developing countries usually rely on supplies from developed 
countries. Simultaneously, the price of those production costs moves along with currency and inflation movements in developing countries. Uncertainty, inflation, and depreciation of the national currencies in developing countries influence the fluctuations in production costs and the income of the firms in those countries. It affects the volatility of the accounting profits earned by firms in developing countries so that the total of the aggregate of accounting earnings cannot act as a reliable predictor of future GDP's growth. This finding is supported by the higher volatility of the aggregate of accounting earnings in developing countries rather than in developed countries.

This economic uncertainty relates to resource allocations, which have not yet been able to create economic value added. Efficient resource allocations are depicted by sophisticated capital markets and active money markets. It is usually known as the strong-form stock exchange in the capital market's hypothesis. Most of the developing countries do not have markets as sophisticated as those found in developed countries. In other words, inefficient allocations cannot contribute to higher economic productivity values. Consequently, low economic productivity causes a null association between the earnings aggregate and future GDP growth.

Secondly, the association between accounting earnings and future GDP growth cannot capture the activity of the real sector economy in developing countries. Moreover, the aggregate of accounting earnings is not able to capture the earnings from private firms and Small to Medium Enterprises (SMEs). For example, in Indonesia, there are about 47,000 private companies, and the number of SMEs is around 12,000. The total accounting earnings of these private firms and SMEs should be higher than the earnings obtained from the 496 listed firms in 2015.

The points mentioned above occur in reverse in the capital markets in developed countries. It means that the aggregate of the accounting earnings properties in the developing countries is different from that in the developed ones. Those differential properties are the informativeness level at the market-wide and nation-wide levels. This study, therefore, concludes that those properties could indicate the practical benefits in emerging capital markets. It means that accounting earnings' properties in the developed countries could capture most of the economic sectors within those countries with efficient resource allocations. However, this does not occur in developing countries.

Finally, most developing countries do not have an integrated national administration and accounting system to recognize and to record income productivity, as well as consumption, from all the economic sectors. This finding relates to the second finding of this study. Developed countries possess formal economic systems. Meanwhile, developing countries do not have such systems in place yet. In other words, the developing countries are dominated by their informal sectors. Furthermore, these informal sectors are not recorded and recognized as part of the total GDP. Simultaneously, the aggregate of accounting earnings cannot record and recognize them either. Therefore, GDP, as well as accounting earnings, cannot act primarily to assess the performance activities or economic productivity of a country. On the other hand, the developed countries can record and to recognize or formalize their underground economic productivity.

\section{CONCLUSIONS, LIMITATIONS AND FUTURE RESEARCH}

This study examines the association between the aggregate accounting earnings and future GDP's growth in 39 Asia-Pacific and African countries, covering the period from 1989 to 2015. It finds that the aggregate accounting earnings are only informative of future GDP growth in the eight developed countries whose earnings growth is positive. This research donates contributions to the existing literature by extending the research's scope from the United States (Konchitchki \& Patatoukas, 2014) to an international setting. This study concludes that the aggregate of accounting earnings is not a reliable or 
good predictor for future GDP growth in developing countries. This conclusion is based on the test results after partitioning the sample into Asia-Pacific and African country groups. Moreover, it is also still supported after this study undertook a yearly basis periodical sensitivity test. Nevertheless, this study finds no evidence in the periods of 1989-1996, 2000-2007, and 2010-2015 that changes in accounting earnings are not a reliable predictor for future GDP's growth in the developing countries. However, it is only an incidental finding.

Aside from that incidental finding, this study concludes that developing countries in the three periods experienced economic uncertainty as there were few economic changes. Additionally, this study identifies the possibilities that caused the unsupported evidence of the objectives of this study. It argues that accounting numbers do not adequately reflect economic productivity in developing countries, for several reasons: Firstly, most developing countries have more considerable future economic uncertainties relative to those of the developed countries. These economic uncertainties are related to resource allocations that are not able to create added economic value. Secondly, the accounting numbers and GDP cannot capture the real economic sectors in the developing countries. Furthermore, the aggregate of the accounting numbers does not capture most of the private firms' and small firms' profits that contribute to economic activity. Lastly, most of the developing countries do not have an adequate national administrative and integrated accounting systems to recognize and to record all of the income from production activities, as well as the consumption from all their economic sectors.

The three possibilities mentioned above come from the internal economic climates of developing countries. This study identifies economic-political and sociocultural factors that cause the null association between the aggregate accounting earnings and the future GDP's growth. Those factors are as follows: There are so many costly power seizures in developing countries. Consequently, they severely affect economic productivity, which leads to low GDP growth.

Economic and sociocultural factors also contribute to the null association between the aggregate of accounting earnings and the future GDP's growth. Low education levels usually characterize in developing countries. The low education then contributes to low economic and sociocultural abilities. Low education levels limit the social needs to only physiological and self-security. If the country has fulfilled both needs, social needs usually stop at this point. On the other hand, social needs are not only limited to physiological and self-security but also extended to self-esteem and self-genuine actions. This sociocultural distinction between the developed and the developing countries leads to different GDP numbers and growth.

\subsection{Limitations}

This study has some limitations. Firstly, it does not consider the basic concept of GDP's measurement. GDP focuses on the cost of the goods sold while accounting earnings measure the added economic value as a result of retaining a firm's capital, which already excludes the cost of the goods sold. The distinction concept that underlies these two measurements leads to the total figure for GDP is a multiplier effect for the cost of the goods sold. Secondly, accounting earnings are an added value to the private sector. This study does not include government expenditure as next year's GDP growth predictor. Nevertheless, this study rejects the notion that the total profits of private firms fund most government expenditure. Similarly, state-owned companies' profits could be incidentally joined with private firms' profits. However, this study could not identify the magnitude of this joint productivity.

Thirdly, most of the developing countries have different accounting standards for different sectors. For example, general accounting standards are for firms listed on the capital markets, and shariah accounting standards are for firms with a sharia basis. This condition shows that one single set of accounting standards does not produce the aggregate of the accounting earnings. The heterogeneity of the 
accounting standards leads to the inability of the earnings aggregate to predict macroeconomic activities regarding the relative earnings aggregate produced by the developed countries, which generally comes from one single set of accounting standards. Fourthly, most of the GDP calculations in developing countries is still done using a multi-sector approach, so that the calculated GDP is not the actual, real figure. For example, the informal sectors in developing countries do not record their production costs, as well as their income from other entities. Similarly, developed countries may recognize and record their underground economies into their GDP figures, but this goes unrecorded by the developing countries.

Fifthly, most developing countries usually have low levels of investor protection, weak law enforcement, less-developed capital markets, poor information environments, and low quality of accounting information (Porta et al., 1998; Rafael et al., 2006). Furthermore, country-level accounting information' quality would impact the predictive power of the aggregate accounting information for GDP's growth (Leuz et al., 2003). It would probably impact the validity of this research's conclusions too.

\subsection{Future study}

According to the conclusions and limitations, this study formulates several possible future studies to complement this study. Firstly, not all developed countries use the same accounting standards, such as European countries adopting IFRS, and the US adopted its accounting standards (US GAAP_SFAC/S). Prior studies show that IASB-IFRS and US GAAP-SFAC/S produce significantly different figures and results. Therefore, future studies may examine the reliability or accuracy of the aggregate of accounting earnings produced by IFRS and SFAC/S. Future studies should be able to formulate a statisticalconclusion validity for use as next year's GDP growth predictor.

Secondly, due to the inability of this study to support the association between the aggregate of accounting earnings and the GDP's growth, this study opens up to using any possible factors which can replace accounting earnings. The first possibility is employing total assets and changes in total assets. The change is a sensible option as assets, along with their growth, refer to the capital maintenance concept. The second possibility is to use debt and its changes, as well as equity capital and its changes. These possibilities show a firm's scalability. The other possibility is replacing the aggregate of accounting earnings with total costs, as they are matched against revenue in the income statement. Thirdly, the validity of GDP's growth $\left(\mathrm{g}_{\mathrm{it}+1}\right)$ in the developing countries is called into doubt. Thus, the GDP growth employed in a future study might be modified into $\left(g_{i t+1)}\right.$ - (eit+1.git+1), or another modified GDP growth, which has high validity for its measurement.

\section{ACKNOWLEDGMENT}

I am grateful to the Faculty of Economics and Business, the University of Gadjah Mada, in supporting and financing all research activities up to its reporting. This study was funded by the Public Fund for Research Programs of the fiscal year 2015. The author has presented this article at a research seminar at the Faculty of Economics and Business, UGM, on December 19th, 2016, and the Indonesian Accounting Symposium on August 23-26th, 2016. The author would like to convey his gratitude to any critics and for comments and suggestions from all the seminars' participants. 


\section{REFERENCES}

A. Sims, Christopher. (2002). The Role of Models and Probabilities in the Monetary Policy Process (Vol. 66).

Abarbanell, Jeffery S. (1991). Do analysts' earnings forecasts incorporate the information in prior stock price changes? Journal of Accounting and Economics, 14(2), 147-165. DOI: https://doi.org/10.1016/01654101(91)90003-7

Ang, Andrew, Bekaert, Geert, \& Wei, Min. (2007). Do macro variables, asset markets, or surveys forecast inflation better? Journal of Monetary Economics, 54(4), 1163-1212. DOI: https://doi.org/10.1016/j.jmoneco.2006.04.006

Ang, Andrew, Piazzesi, Monika, \& Wei, Min. (2006). What does the yield curve tell us about GDP growth? Journal of Econometrics, 131(1), 359-403. DOI: https://doi.org/10.1016/j.jeconom.2005.01.032

Baghestani, Hamid, \& M Kianian, Amin. (1993). On the Rationality of U.S. Macroeconomic Forecasts: Evidence from a Panel of Professional Forecasters (Vol. 25).

Ball, Ray, Sadka, Gil, \& Sadka, Ronnie. (2009). Aggregate earnings and asset prices. Journal of Accounting Research, 47(5), 1097-1133.

Basu, S. (1977). Investment Performance of Common Stocks in Relation to Their Price-Earnings Ratios: A Test of The Efficient Market Hypothesis. The Journal of Finance, 32(3), 663-682. doi: 10.1111/j.15406261.1977.tb01979.x

Beaver, William H. (1981). Financial reporting: an accounting revolution: Prentice-Hall.

Becker, Gary S., Philipson, Tomas J., \& Soares, Rodrigo R. (2005). The Quantity and Quality of Life and the Evolution of World Inequality. American Economic Review, 95(1), 277-291. DOI: 10.1257/0002828053828563

Bradshaw, Mark T., Drake, Michael S., Myers, James N., \& Myers, Linda A. (2012). A re-examination of analysts' superiority over time-series forecasts of annual earnings. Review of Accounting Studies, 17(4), 944-968. DOI: $10.1007 / \mathrm{s} 11142-012-9185-8$

Brown, Philip, \& Ball, Ray. (1967). Some Preliminary Findings on the Association between the Earnings of a Firm, Its Industry, and the Economy. Journal of Accounting Research, 5, 55-77. DOI: 10.2307/2489908

Bureau of Economic Analysis. (2002). Corporate Profits: Methodology Paper. United States Department of Commerce, Economics and Statistics Administration, Bureau of Economic Analysis.

Bureau of Economic Analysis. (2004). Corporate Profits in The GDP Accounts. BEA paper series, No. 0040.United States Department of Commerce, Economics and Statistics Administration, Bureau of Economic Analysis.

Bureau of Economic Analysis. (2007). Measuring the Economy: A Primer of GDP and the National Income and Product Accounts. United States Department of Commerce, Economics and Statistics Administration, Bureau of Economic Analysis.

Chung, Richard, \& Kryzanowski, Lawrence. (1999). Accuracy of consensus expectations for top-down earnings per share forecasts for two S\&P indexes. Applied Financial Economics, 9(3), 233-238. DOI: 10.1080/096031099332302

Clarida, Richard, Gali, Jordi, \& Gertler, Mark. (2000). Monetary Policy Rules and Macroeconomic Stability: Evidence and Some Theory. The Quarterly Journal of Economics, 115(1), 147-180.

Conrad, Jennifer, Cornell, Bradford, \& Landsman, Wayne. (2000). When is Bad News Really Bad News (Vol. 57).

Darrough, Masako. (2002). A Positive Model of Earnings Forecasts: Top-Down versus Bottom-Up (Vol. 75).

Dechow, Patricia M., Kothari, S. P., \& L. Watts, Ross. (1998). The relation between earnings and cash flows. Journal of Accounting and Economics, 25(2), 133-168. DOI: https://doi.org/10.1016/S0165-4101(98)00020-2

Fama, Eugene F. (1981). Stock Returns, Real Activity, Inflation, and Money. The American Economic Review, 71(4), 545565.

Fischer, Stanley, \& Merton, Robert. (1984). Macroeconomics and Finance: The Role of the Stock Market (Vol. 21).

Fleurbaey, Marc. (2009). Beyond GDP: The Quest for a Measure of Social Welfare. Journal of Economic Literature, 47(4), 1029-1075. DOI: $10.1257 /$ jel.47.4.1029

Fleurbaey, Marc, \& Gaulier, Guillaume. (2009). International comparisons of living standards by equivalent incomes. Scandinavian Journal of Economics, 111(3), 597-624.

Gonedes, Nicholas J. (1973a). Evidence on the information content of accounting numbers: Accounting-based and market-based estimates of systematic risk. Journal of Financial and Quantitative Analysis, 8(3), 407-443. 
Gonedes, Nicholas J. (1973b). Properties of accounting numbers: Models and tests. Journal of Accounting Research, 212237.

Harvey, Campbell R. (1989). Forecasts of economic growth from the bond and stock markets. Financial Analysts Journal, 45(5), 38-45.

Henderson, J. Vernon, Storeygard, Adam, \& Weil, David N. (2012). Measuring Economic Growth from Outer Space. American Economic Review, 102(2), 994-1028. DOI: 10.1257/aer.102.2.994

Ho, Yew-Kee, \& Sequeira, John M. (2007). Earnings Surprises, Asymmetry of Returns, and market-level Changes: An Industry Study. Journal of Accounting, Auditing \& Finance, 22(1), 29-55. DOI: 10.1177/0148558X0702200104

Karnitis, G., \& Karnitis, E. (2017). Sustainable growth of EU economies and Baltic context: Characteristics and modeling. Journal of International Studies, 10(1), 15.

Kieso, Donald E, Weygandt, Jerry J, \& Warfield, Terry D. (2010). Intermediate Accounting: IFRS edition (Vol. 2): John Wiley \& Sons.

Konchitchki, Yaniv. (2011). Inflation and Nominal Financial Reporting: Implications for Performance and Stock Prices (Vol. 86).

Konchitchki, Yaniv, \& Patatoukas, Panos N. (2014). Accounting earnings and gross domestic product. Journal of Accounting and Economics, 57(1), 76-88. DOI: https://doi.org/10.1016/j.jacceco.2013.10.001

Kothari, S. P. (2001). Capital markets research in accounting. Journal of Accounting and Economics, 31(1), 105-231. DOI: https://doi.org/10.1016/S0165-4101(01)00030-1

Kothari, S. P., Lewellen, Jonathan, \& Warner, Jerold B. (2006). Stock returns, aggregate earnings surprises, and behavioral finance. Journal of Financial Economics, 79(3), 537-568. DOI: https://doi.org/10.1016/i.jfineco.2004.06.016

Landefeld, J. Steven, Seskin, Eugene P., \& Fraumeni, Barbara M. (2008). Taking the Pulse of the Economy: Measuring GDP. Journal of Economic Perspectives, 22(2), 193-216. DOI: 10.1257/jep.22.2.193

Leuz, Christian, Nanda, Dhananjay, \& Wysocki, Peter D. (2003). Earnings management and investor protection: an international comparison. Journal of Financial Economics, 69(3), 505-527. DOI: https://doi.org/10.1016/S0304405X(03)00121-1

Lev, Baruch, \& Nissim, Doron. (2004). Taxable Income, Future Earnings, and Equity Values. The Accounting Review, 79(4), 1039-1074. DOI: 10.2308/accr.2004.79.4.1039

Lopez, Thomas, \& L. Rees, Lynn. (2001). The Effect of Meeting Analyst Forecasts and Systematic Positive Forecast Errors on the Information Content of Unexpected Earnings. Working Paper (Vol. 17).

McCloskey, Deirdre. (1993). Other Things Equal: Schelling's Five Truths of Economics. Eastern Economic Journal, 19(1), 109-111.

Newey, Whitney K., \& West, Kenneth D. (1987). A Simple, Positive Semi-Definite, Heteroskedasticity, and Autocorrelation Consistent Covariance Matrix. Econometrica, 55(3), 703-708. DOI: 10.2307/1913610

Oliinyk, V., \& Kozmenko, S. (2019). Forecasting and management of the gross domestic product. Journal of International Studies, 12(4), 14.

Patatoukas, Panos N. (2014). Detecting news in aggregate accounting earnings: implications for stock market valuation. Review of Accounting Studies, 19(1), 134-160. DOI: 10.1007/s11142-013-9221-3

Porta, Rafael La, Florencio Lopez-de-Silanes, Andrei Shleifer, \& Robert W. Vishny. (1998). Law and Finance. Journal of Political Economy, 106(6), 1113-1155. DOI: 10.1086/250042

Rafael, Porta, Florencio, Lopez-De-Silanes, \& Andrei, Shleifer. (2006). What Works in Securities Laws? The Journal of Finance, 61(1), 1-32. doi: 10.1111/j.1540-6261.2006.00828.x

Romer, Christina D., \& Romer, David H. (2000). Federal Reserve Information and the Behavior of Interest Rates. American Economic Review, 90(3), 429-457. DOI: 10.1257/aer.90.3.429

Shevlin, Terry. (1990). Estimating corporate marginal tax rates with asymmetric tax treatment of gains and losses. Journal of the American Taxation Association, 12(1), 51-67.

Shkolnyk, I., Kozmenko, S., Kozmenko, O., \& Mershchii, O. (2019). The impact of the economic financialization on the level of economic development of the associate EU member states. Economics and Sociology, 12(4), 15. 
Skinner, Douglas J., \& Sloan, Richard G. (2002). Earnings Surprises, Growth Expectations, and Stock Returns or Don't Let an Earnings Torpedo Sink Your Portfolio. Review of Accounting Studies, 7(2), 289-312. DOI: 10.1023/a:1020294523516

Sumiyana, Sumiyana, Atmini, Sari, \& Sugiri, Slamet. (2019). Predictive power of aggregate corporate earnings and their components for future GDP growths: An international comparison. Economics and Sociology, 12(1), 18. DOI: DOI:10.14254/2071-789X.2019/12-1/7

Taylor, John B. (1993). Discretion versus policy rules in practice. Paper presented at the Carnegie-Rochester conference series on public policy.

Tung, L. T. (2018). Impact of remittance inflows on the trade balance in developing countries. Economics \& Sociology, 11(4), 15.

White, Halbert. (1980). A Heteroskedasticity-Consistent Covariance Matrix Estimator and a Direct Test for Heteroskedasticity. Econometrica, 48(4), 817-838. DOI: 10.2307/1912934

Zarnowitz, Victor, \& Braun, Phillip. (1994). Twenty-Two Years of the NBER-ASA Quarterly Economic Outlook Surveys: Aspects and Comparisons of Forecasting Performance. 